\title{
Aplicación del análisis estructural de prospectiva al diseño de estrategias de desarrollo rural: el caso de la comarca de Jerez
}

MATEO AMBROSIO-ALBALÁ

Unidad de Cooperación Financiera Reembolsable (Programa AECID - FIIAPP), Agencia Española de Cooperación Internacional al Desarrrollo (AECID), ESPAÑA. E-mail: mateo.ambrosio@fiiapp.org

JOSÉ M. MARTÍN LOZANO

Departamento de Gestión Empresarial y Métodos Cuantitativos, UNIVERSIDAD DE CÓRDOBA, ESPAÑA. E-mail: jmmartin@etea.com

\section{PEDRO P. PÉREZ HERNÁNDEZ}

Departamento de Economía General, Ciencias Jurídicas y Sociología, UNIVERSIDAD DE CÓRDOBA, ESPAÑA. E-mail: ppperez@etea.com

\section{RESUMEN}

Con la aprobación del Reglamento (CE) 1698/2005 del Consejo de la Unión Europea (UE) de 20 de septiembre de 2005 se puso en marcha el cuarto programa de ayuda al desarrollo rural para el período 2007-2013. Son varias las regiones españolas afectadas por este reglamento, entre ellas Andalucía. En este artículo, se expone de manera sintética el enfoque metodológico utilizado para la Nueva Estrategia Rural para Andalucía (NERA) del gobierno de la comunidad y con mayor amplitud la aplicación del Análisis Estructural de Prospectiva (AEP). El AEP es un método idóneo para analizar la complejidad de elementos, factores e interacciones presentes en los territorios rurales, así como para entender las variables en su situación presente y futura. Para ver la bondad de esta metodología se presentan los resultados obtenidos una vez aplicada a la comarca de Jerez. Finalmente se concluye el análisis con la discusión de los resultados y el análisis de las fortalezas y debilidades de la metodología aplicada.

Palabras clave: Desarrollo Rural, Planificación Estratégica, Análisis Estructural de Prospectiva.

\section{An Application of the Prospective Structural Analysis to the Design of Rural Development Strategies: the Case of the Xerez County}

\begin{abstract}
The Council Regulation (EC) 1698/2005 approved on $20^{\text {th }}$ September 2005 was the starting point of the fourth programme on support for rural development for the period 2007-2013. There are several Spanish regions covered by this regulation, Andalusia is one of them. This paper presents in a synthetic way the methodological approach followed to design the New Rural Strategy for Andalusia (NERA, acronym in Spanish) of the Andalusian regional government and more broadly the use of the Prospective Structural Analysis (PSA) technique. This research suggests that the PSA is a suitable method to analyse the complexity of elements, factors and their interactions in the rural areas, as well as to understand variables in their present and future situation. It has been implemented in Jerez local area and its results are shown. After discussing the results, conclusions have been drawn. Finally the strengths and weaknesses of this methodology have been studied.
\end{abstract}

Keywords: Rural Development, Strategic Planning, Prospective Structural Analysis.

Clasificación JEL: R11, R58

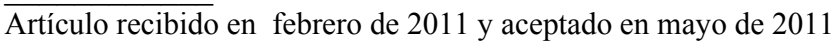

Artículo disponible en versión electrónica en la página www.revista-eea.net, ref. ə-29115 


\section{INTRODUCCIÓN}

El desarrollo rural nace en Europa de forma nítida, como proceso sistematizado e instrumentalizado a través del sector público, con la publicación de El futuro del mundo rural (Comisión Europea, 1988) y con la reforma de los fondos estructurales para adaptarlos a las exigencias internas -mayor cohesión económica y social ${ }^{1}$ - y externas -negociaciones en el ámbito de la Ronda Uruguay. Este cambio fundamental de orientación en la todopoderosa política agraria común, se vio plasmado en 1991 con la Iniciativa LEADER (Delgado, Ramos y Pretel, 2007) cuyo objetivo principal fue la promoción y el desarrollo económico de las zonas rurales más desfavorecidas de los entonces Estados miembros de la Unión Europea, aplicando para ello el enfoque territorial y participativo (Sumpsi Viñas, 2006). A lo largo de estos años dicha iniciativa se ha ido consolidando mediante la puesta en funcionamiento de sucesivas ediciones $^{2}$ y el reconocimiento expreso del interés del enfoque de trabajo, incorporado como eje metodológico en la última propuesta reglamentaria de la Unión Europea para el período marco 2007-2013.

La puesta en marcha de tal iniciativa supuso la creación de una red de grupos de acción local (GAL) en cada una de las regiones consideradas Objetivo 1 y $5 \mathrm{~b}$ de los Estados miembros. Con el paso del tiempo, en algunas comunidades autónomas como Andalucía, estos grupos pasaron a denominarse Grupos de Desarrollo Rural (GDR). El objetivo de estos grupos estuvo claro desde su creación (Comisión Europea, 1991) y han sido ellos los que han promovido el proceso de planificación y promoción del desarrollo (González Fernández, 2006) dinamizando a los distintos agentes económicos y sociales de las zonas rurales desde que comenzó esta iniciativa en 1991 y al menos hasta 2013.

En línea con el Reglamento (CE) 1698/2005 del Consejo de la Unión Europea (UE) relativo a la ayuda al Desarrollo Rural a través del Fondo Europeo Agrario de Desarrollo Rural (FEADER), el gobierno autonómico andaluz impulsó la elaboración de una Nueva Estrategia Rural para Andalucía (NERA). Siguiendo la lógica ascendente propia del enfoque LEADER, esta nueva estrategia debía agregar a escalas provincial y autonómica las demandas y visiones de desarrollo de los GAL comarcales, a la vez que brindar elementos de análisis relevantes para que dichos GAL diseñaran líneas de acción para sus territorios. Una premisa y dos metas con implicaciones metodológicas considerables para llevar a cabo el ejercicio de planificación estratégica, en especial, en términos de coordinación (dada la cantidad de GAL existentes en

\footnotetext{
${ }^{1}$ Sobre el coste de la cohesión en Europa ver García Nicolás (2009).

${ }^{2}$ LEADER II y LEADER +. Para España a la iniciativa LEADER hay que unirle desde 1995 el Programa Operativo para el Desarrollo y la Diversificación Económica de Zonas Rurales (PRODER) de aplicación exclusiva para determinadas regiones de nuestro país.
} 
Andalucía) y de recopilación de información respetando el carácter participativo y deliberativo propio del enfoque LEADER.

El reto pues afectaba no sólo al enfoque metodológico global, sino también al propio método para recabar información primaria. En la propuesta metodológica se optó por aplicar el método del Análisis Estructural de Prospectiva (AEP). El AEP es un método habitual de los estudios de prospectiva para la elaboración de escenarios. Permite identificar las relaciones de influencia entre variables de un sistema dado y señalar qué papel tienen en su evolución. Una de las principales ventajas del AEP es que contribuye a la acción por medio de la deliberación estructurada, de ahí su valor para la planificación estratégica.

El objeto de este artículo es exponer y valorar la aplicación del método del AEP al diseño de estrategias de desarrollo en comarcas rurales. El artículo se ha dividido en cuatro partes. En la primera se exponen las dificultades que conlleva analizar una zona rural en coherencia con el enfoque territorial de LEADER y se describe a grandes rasgos el método del AEP. A continuación, se describe la metodología global del proyecto que se aplicó a las cincuenta y una comarcas andaluzas y la metodología del AEP usada en el proyecto NERA. Seguidamente se discuten los resultados de la aplicación del análisis de prospectiva a una comarca concreta, Jerez, y finalmente se exponen las conclusiones obtenidas en la aplicación del AEP cuyos resultados deberán ser contrastados en el futuro con la realidad del desarrollo rural, al concluir el periodo marco de actuación en esta comarca.

\section{MÉTODOS INTERPRETATIVOS PARA EL ANÁLISIS DEL TERRITORIO RURAL}

\subsection{El análisis del territorio como "dilema a construir"}

Uno de los principales leit motiv del enfoque territorial en las políticas rurales es involucrar a los agentes rurales en la elaboración e implementación de estrategias y planes de desarrollo para sus zonas. La interpretación que hacen los agentes locales sobre las restricciones, potenciales e incentivos para el cambio en sus comunidades sirven como inputs fundamentales para definir el territorio deseado. De ahí que el territorio rural sea contemplado como una construcción social. Una vía para estudiar el territorio rural es pues indagar en el proceso de deliberación que lo origina.

El territorio rural es un concepto complejo, sistémico y dinámico que emerge de la interacción de una multiplicidad de factores, presentes a diferentes escalas y niveles de análisis (Ambrosio-Albalá y Bastiaensen, 2010). El estudio del territorio rural así entendido requiere no sólo un enfoque multidisciplinar propio de su naturaleza sistémica y de los múltiples elementos que lo integran. Son 
necesarias además herramientas de análisis que permitan considerar las interrelaciones entre dichos elementos (Habegger, 2010).

Habitualmente, el estudio de las regiones o territorios se realiza mediante la explotación de datos e información estadística al uso. En esta línea, los modelos de contabilidad social ayudan a identificar relaciones estructurales para economías concretas (De Miguel y Manresa, 2004; Llop Llop, 2007). Cuando se sigue un enfoque sistémico, es frecuente la elaboración de modelos formales, eminentemente cuantitativos, en línea con la teoría general de sistemas y la dinámica de sistemas (von Bertalanffy, 1968; Forrester, 1961). Amén de la dificultad insoslayable de obtener los datos necesarios a escala comarcal, estos métodos presentan algunas limitaciones para su aplicación en sistemas sociales (Rosenhead, 1996). En las últimas décadas, el campo del pensamiento sistémico ha ido incorporado progresivamente nuevas áreas de interés, relacionadas con el diálogo y el aprendizaje interactivo (Schlange and Jüttner, 1997). Desde esta perspectiva cognitiva, la construcción social de un territorio rural puede considerarse un "dilema a construir" ("unstructured problem"), en la medida que interactúan múltiples actores y perspectivas y surgen conflictos de interés y de interpretación que dificultan la elaboración de un objeto de análisis acotado y único. Para resolver este problema de construcción del objeto de análisis se necesitan herramientas de análisis que organicen el pensamiento y las deliberaciones colectivas, a la par que ayudan a generar una perspectiva compartida sobre un mismo problema (Bolanos et al., 2005). A efectos prácticos, este tipo de herramientas deben ser accesibles para agentes con diferentes capacidades y perspectivas, facilitar el intercambio de opiniones entre ellos y trabajar de forma iterativa a medida que se producen las deliberaciones (Mingers y Rosenhead, 2004).

Existe un amplio abanico de técnicas que responden a estos criterios. Así están los llamados métodos para estructurar problemas ("problem-structuring methods", PSM) (Rosenhead, 1996), tales como el mapeo cognitivo ("cognitive mapping") (Axelrod, 1976) y los mapas cognitivos difusos ("fuzzy cognitive maps") (Kosko 1986). Estas técnicas están especialmente indicadas en contextos donde se requiere un amplia participación. No requieren el uso de un aparato matemático muy sofisticado, aunque se pueden usar ordenadores para procesar los resultados. Un poco más complejos y basados en el uso de programas informáticos se encuentran las llamadas técnicas de modelado estructural ("structural modelling") (Lendaris, 1980), tales como el modelado estructural interpretativo ("interpretive structural modelling", ISM) (Warfield, 1974; Janes, 1998) y el método de impactos cruzados ("cross-impact methods") (Weimer-Jehle, 2006). Estos últimos encuentran una aplicación fundamental en el campo de la prospectiva estratégica y la elaboración de escenarios (Godet, 1993; Coates, 2000). En este campo, una de las técnicas más conocidas es el Análisis Estructural de Prospectiva (AEP). 


\subsection{El Análisis Estructural de Prospectiva (AEP)}

El AEP es un método que se utiliza habitualmente en la construcción de escenarios y el diseño de futuros, un subconjunto del campo de la prospectiva estratégica. Este campo de estudios asume que el futuro no surge de extrapolar tendencias en el pasado, pero se puede construir definiendo escenarios deseables y viables e impulsando las acciones necesarias para alcanzarlos (Gavigan y Scapolo, 2001; Godet, 1985, 1993, 2001). Esta construcción del futuro básicamente se sirve de técnicas para estructurar procesos de deliberación colectiva, describir situaciones en el presente y elaborar visiones a largo plazo (Gertler and Wolfe, 2004).

El método del AEP ayuda a describir un sistema identificando las relaciones de influencia (en lugar de las relaciones de causa-efecto) entre los elementos integrantes del sistema. Esta descripción se lleva a cabo por medio de un proceso de reflexión y deliberación colectiva en el que se rellena una matriz de doble entrada. Mediante la aplicación de las propiedades de las cadenas de Markov, esta herramienta permite generar unas jerarquías y unas clasificaciones de los elementos conforme a sus propiedades de motricidad (influencia que un elemento ejerce sobre otro) y dependencia (influencia que un elemento recibe de otro). De esta forma, el método pone de relieve la estructura de relaciones entre las variables del sistema y señala cuáles son las variables esenciales en su evolución.

\subsection{Aplicaciones previas del AEP}

En la última década la prospectiva y la creación de escenarios han ido ganado aceptación y difusión como herramientas de análisis estratégico regional en Europa (Cariola and Rolfo, 2004). Además de las experiencias recogidas en revistas especializadas (Kelly, 2004; Grossmann, 2006), la web de la Red Europea de Seguimiento Prospectivo (European Foresight Monitoring Network, EFMN) recoge numerosas experiencias a escala regional ${ }^{3}$.

En lo que se refiere a la aplicación a zonas rurales y sectores económicos en localidades específicas, el uso de la prospectiva y el diseño de escenarios también resulta de interés. Así sucede con el diseño de escenarios futuros para zonas rurales en Europa (Kinsella et al., 2005; Mahroum, 2007). Para el sector agrícola en España, son dignos de mención los estudios sobre la situación de la agricultura en Castilla y León (Gómez-Limón et al., 2009) y a nivel nacional (Gómez-Limón y Gómez-Ramos, 2008). En este trabajo, los autores subrayan que el trabajo realizado debe servir, de manera especial, para orientar a las administraciones públicas en el establecimiento de las líneas políticas básicas que permitan el desarrollo integral del sector agrario y del mundo rural.

\footnotetext{
${ }^{3}$ http://www.efmn.info/ [Último acceso: 29 abril 2011]
} 
También existen aplicaciones sectoriales en Latinoamérica. Un caso es la aplicación de la prospectiva, como herramienta de planificación estratégica, para el estudio del aceite de palma y de palmiste en Colombia (Jaimes et al., 2003). Esta aplicación permitió establecer a los autores las directrices para el desarrollo del sector oleoquímico a largo plazo. Otra aplicación se realizó para el sector turístico de una zona de escasos recursos pero con una gran proyección turística en la región de la Araucania (Chile) (Vergara y Arrais de Souza, 2005). Los autores indican que el uso de la metodología AEP resultó muy potente para establecer planes de futuro en dicha región. El análisis de las motricidades y dependencias entre variables regionales les permitió agruparlas y establecer escenarios de futuro para el turismo de la región hasta el año 2012.

El uso del AEP como método para el análisis de territorios está posiblemente menos extendido, pero existen experiencias reseñables. Tal es el caso del estudio del ordenamiento territorial en la provincia de Cienfuegos (Cuba) con el objetivo de determinar variables y actores clave para las acciones y estrategias territoriales de la citada provincia (Sarria et al., 2009). En esta investigación se utilizaron 32 macrovariables y como resultado de la aplicación del AEP se obtuvo una adecuada jerarquización de éstas que permitió priorizar las acciones estratégicas para el ordenamiento del territorio.

En lo que respecta al desarrollo rural, destaca un estudio reciente (AmbrosioAlbalá y Delgado, 2008) en el que se presentó un análisis comparativo de la realidad territorial de dos comarcas distintas correspondientes a dos regiones situadas en países diferentes, España y Nicaragua. Los autores concluyen que esta metodología es fundamental para construir el territorio rural como resultado de un proceso de reflexión realizado por los actores del mismo. Igualmente, señalan que en el proceso de comparación entre dos territorios que se encuentran en diferentes momentos de desarrollo, se observaron determinadas similitudes en los modelos adoptados.

\section{LA NUEVA ESTRATEGIA RURAL PARA ANDALUCÍA (NERA)}

La aprobación del Reglamento (CE) 1698/2005 para el Fondo Europeo Agrario de Desarrollo Rural (FEADER) ha marcado un importante punto de inflexión en la política de desarrollo rural de la UE. Dicho Reglamento constituye el principal instrumento para la política rural en el período 20072013.

Por su parte, el día 20 de febrero de 2006 se aprobó la Decisión del Consejo relativa a las Directrices Estratégicas comunitarias de Desarrollo Rural para dicho período. Siguiendo la disposición prevista en el artículo 9 del Reglamento 
(CE) $1698 / 2005^{4}$, las Directrices fijaron las prioridades en materia de desarrollo rural en función de los ejes y objetivos del citado Reglamento, reflejando el papel socioeconómico y ambiental de la actividad agraria y las actuaciones prioritarias en relación con los objetivos de desarrollo sostenible de la Cumbre de Gotemburgo y la estrategia de crecimiento y empleo de la Cumbre de Lisboa.

Esta nueva normativa fue aplicada en cada una de las comunidades autónomas españolas afectadas. En Andalucía, la Consejería de Agricultura y Pesca la puso en marcha mediante la elaboración de una Estrategia de Desarrollo Rural para Andalucía en el marco de una planificación estratégica de la política agraria andaluza para el periodo 2007-2013. A raíz de esta iniciativa la Consejería andaluza propuso para todos los GAL la homogeneización de la metodología a aplicar, lo que suponía realizar un proyecto, pionero en Europa, que se denominó Nueva Estrategia Rural para Andalucía (NERA) (Junta de Andalucía, 2008). Ha sido la primera vez que se configuró una propuesta estratégica de manera coordinada para los $51 \mathrm{GAL}$ que forman parte de la región.

El proyecto NERA ha fomentado la participación de la sociedad rural y los agentes sociales, económicos y políticos en el debate y análisis de la situación actual de las zonas rurales de Andalucía. El proyecto NERA es una estrategia complementaria a la nueva política económica del gobierno andaluz en el marco de la Unión Europea y una ambiciosa apuesta por el desarrollo sostenible de sus espacios rurales y que se enmarca dentro del Programa de Desarrollo Rural Andaluz para el período 2007-2013.

Las aportaciones de este proyecto se podrían resumir en dos:

- La elaboración de 51 planes estratégicos comarcales y 8 planes estratégicos provinciales y un plan estratégico regional.

- Una metodología novedosa, en la que se hizo uso del AEP y cuya aplicación se expone a continuación.

\section{METODOLOGÍA DE LA NERA}

\subsection{El enfoque metodológico global de la NERA}

El principal aspecto destacable del proyecto NERA es su propia metodología de elaboración. El proyecto se propuso para diseñar un plan estratégico de desarrollo rural para el conjunto de la comunidad autónoma andaluza, que fuera

\footnotetext{
${ }^{4}$ Los ejes temáticos son: aumento de la competitividad de los sectores agrario y forestal, mejora del medio ambiente y del entorno rural y calidad de vida en las zonas rurales y diversificación de la economía rural. Además de los tres ejes temáticos, el Reglamento (CE) 1698/2005 incorpora un cuarto eje metodológico relativo al enfoque LEADER, cuya aplicación en los otros tres ejes es discrecional.
} 
el resultado de integrar ocho planes estratégicos provinciales. Así mismo, cada plan provincial se elaboraría integrando los planes estratégicos de cada uno de los GAL existentes en cada provincia ${ }^{5}$. En todo el proceso intervendrían los actores del territorio, ya que la metodología preveía su participación en cada nivel y en distinto grado.

Para anteriores iniciativas europeas de desarrollo rural, cada GAL realizó su propia estrategia, bien por cuenta propia o por medio de terceros y la metodología de planificación también fue específica de cada comarca. Para el proyecto NERA se propuso trabajar con una metodología común, de manera que se facilitara la tarea de integrar los planes estratégicos comarcales en cada plan provincial. Esta metodología común preveía también la intervención de los actores del territorio en todos los niveles a lo largo del proceso. Es un claro ejemplo, de la capacidad del sector privado para dinamizar (Díez y Gutiérrez, 2008) la economía en perfecta armonía con el sector público. Las peculiaridades de este proceso de planificación estratégica implicaban determinados factores que condicionaron claramente la metodología única a utilizar:

- Los planes estratégicos resultantes debían tener una clara componente participativa: ser elaborados de abajo hacia arriba contando con los principales agentes que intervienen en el ámbito rural andaluz.

- No se tenía constancia de modelos o experiencias de planificación estratégica en zonas rurales en la que un plan resultara de la integración de planes en niveles inferiores. Por lo tanto, hubo que diseñar un método ad hoc para NERA.

- La necesidad de integrar planes hasta llegar al nivel autónomico superior y las restricciones de fechas ${ }^{6}$ exigían que todos los grupos elaboraran sus respectivos planes de manera simultánea, lo que supuso un esfuerzo y recursos ingentes para la coordinación.

- Los responsables de elaborar los planes estratégicos a nivel comarcal (gerente del GAL) eran un colectivo heterogéneo, con experiencia dispar en planificación estratégica y con trabajo cotidiano que atender. Fue necesario incrementar la dotación de recursos humanos de los grupos.

Tal y como se muestra en la Figura 1, el proyecto NERA se planteó en línea con el enfoque ascendente o de 'abajo-arriba' impulsado por la iniciativa LEADER, partiendo de los GAL existentes en Andalucía hasta alcanzar el diagnóstico global para la región.

\footnotetext{
${ }^{5}$ Cada grupo está asignado a una provincia determinada, con alguna excepción, como el caso de la comarca de la Alpujarra, granadina y almeriense, que fue asignada a cada una de sus respectivas provincias.

${ }^{6}$ Recordemos que esta iniciativa sería la base sobre la que descansaría la programación 20072013 elaborada por la Consejería de Agricultura y Pesca en materia de desarrollo rural.
} 
Figura 1

Esquema de la metodología global aplicada en el proyecto NERA para la elaboración de planes estratégicos a nivel comarcal, provincial y autonómico

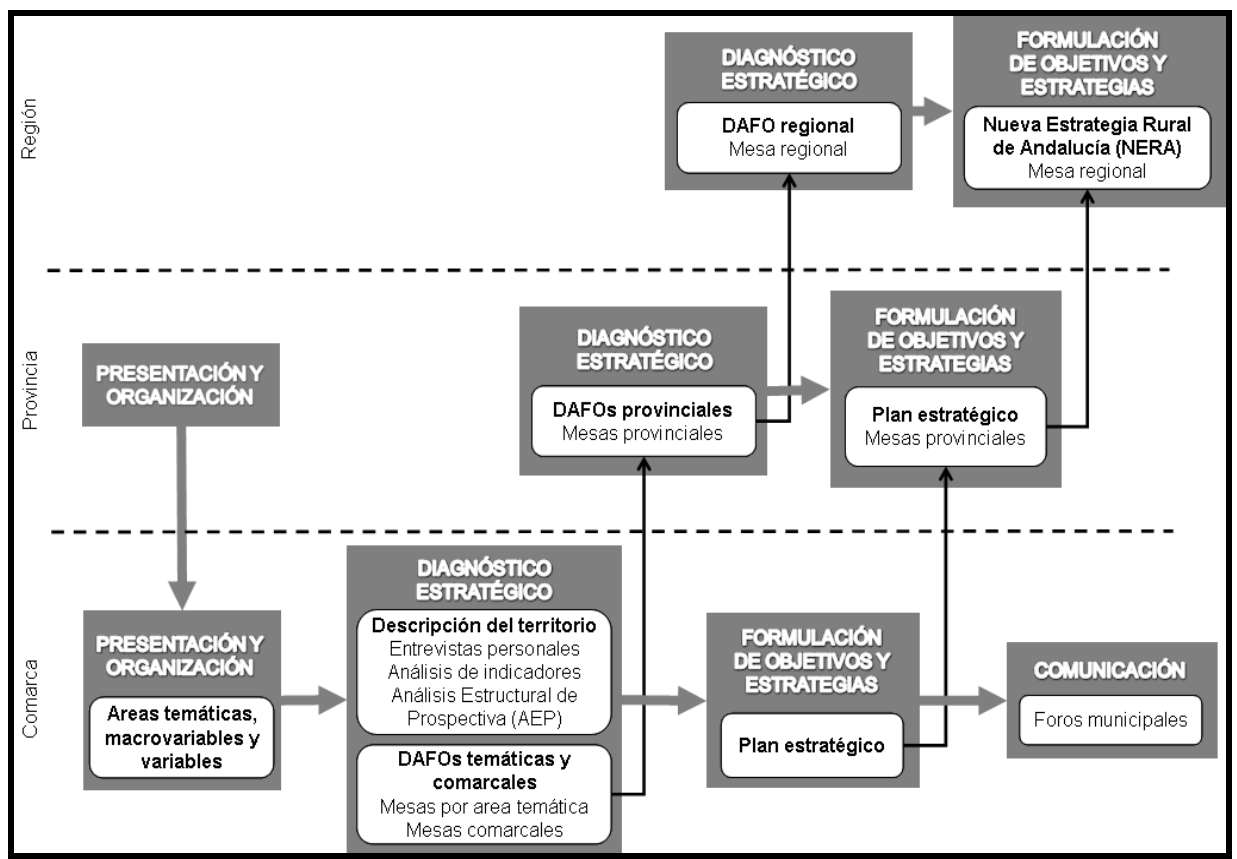

Fuente: Elaboración propia.

Se establecieron tres niveles territoriales de planificación: el comarcal, el provincial y el autonómico o regional. El nivel más complejo fue el primero, fundamento de todo el proceso y sobre el que se apoyan los otros dos.

Desde un punto de vista conceptual, la planificación se estructuró en cinco fases: presentación y organización del proceso, diagnóstico estratégico, definición de objetivos estratégicos, formulación de estrategias y comunicación de resultados. Una vez terminado el proyecto se iniciaron las fases de implantación y control de los respectivos planes.

La presentación y organización del proceso comenzó con la celabración de foros provinciales y comarcales para presentar el proyecto a los agentes del territorio. Se celebraron al inicio del año 2007 y en ellos se consensuó una lista de áreas temáticas, macrovariables y variables para que la aproximación al análisis del territorio en las fases siguientes se hiciera desde las mismas claves. El listado partió de la información disponible en el Sistema de Información Multiterritorial de Andalucía (SIMA) y en las aportaciones realizadas desde las comarcas, según la información disponible en las mismas. 
El diagnóstico estratégico tuvo como objetivo disponer de una valoración participativa de la realidad del territorio. Este diagnóstico consistió en una descripción del territorio y en la elaboración de matrices DAFO por área temática y comarca. La información primaria se obtuvo a lo largo del año 2007 utilizando técnicas al uso: entrevistas, cuestionarios a expertos en aspectos sectoriales del territorio (método Delphi) y mesas de debate y de prospectiva para la aplicación del método AEP en las que participaron agentes locales y expertos en los diferentes temas tratados. Para la obtención de información secundaria a nivel comarcal se consultó el Sistema de Información Multiterritorial de Andalucía (SIMA) y las fuentes propias de instituciones comarcales.

Con el objeto de mantener la homogeneidad de la información primaria, se diseñaron modelos estandarizados de formularios para la recogida de datos de común acuerdo con los distintos agentes (alcaldes, empresarios, representantes de asociaciones, miembros de los GAL, etc.). Así mismo y para facilitar el análisis posterior de los datos obtenidos sobre el territorio, se propuso el diseño y desarrollo de una base de datos según el modelo relacional de datos convencional. Esta base de datos se adjuntó a los documentos finales y a partir de ella se realizaron los oportunos análisis estadísticos y espaciales.

Una vez determinado el diagnóstico comarcal, cada mesa comarcal definió los objetivos de cada plan estratégico, global y por área temática, de forma que aglutinaran las inquietudes de las mesas temáticas. Si era pertinente, se hacía hincapié en algún elemento diferencial de ese territorio.

A esta fase siguió la formulación de las estrategias comarcales. Se consideró necesario conocer qué elementos del territorio tenían capacidad de movilizar o impulsar a otros. Se decidió utilizar las macrovariables como unidad de análisis, en tanto que acotaban claramente la parte del territorio considerado y agregaban en torno a sí a otras variables numerosas pero de menor dimensión. Estas macrovariables fueron objeto de análisis mediante el método AEP. Su aplicación y los resultados obtenidos se describen a continuación.

\subsection{El AEP aplicado al estudio de las comarcas rurales}

El propósito de aplicar el AEP en el proyecto NERA no fue apoyar la elaboración de escenarios, sino detectar macrovariables con capacidad de influir (motrices) o de ser influidas (dependientes). Con esa información los agentes de cada comarca estarían mejor posicionados para diseñar sus estrategias de desarrollo, al interpretar y deliberar de forma conjunta y en profundidad los procesos sociales, económicos, políticos y ambientales que dan forma a la dinámica interna de su sistema territorial.

Con este fin, el AEP se adaptó a la realidad de las zonas rurales a estudiar y su aplicación se estructuró en tres fases: 
- La elaboración del listado de macrovariables.

- La descripción de las relaciones entre las macrovariables.

- La identificación del papel de las macrovariables.

\subsubsection{La elaboración del listado de macrovariables (fase 1)}

El primer paso consistió en elaborar una serie de áreas en las que incluir macrovariables explicativas. Las áreas consideradas fueron seis: Medio físico y Medio ambiente, Población y Sociedad, Infraestructuras y Transporte, Economía, Organización Administrativa y Entorno. A continuación, se consensuó con los cincuenta y un GAL una lista de macrovariables, definidas como constructos $^{7}$, que se muestra en la Tabla 1.

Tabla 1

Relación de áreas temáticas, macrovariables y variables

\begin{tabular}{|c|c|c|}
\hline Macrovariable & Abreviatura & Variables \\
\hline \multicolumn{3}{|c|}{ ÁREA TEMÁTICA 1: MEDIO FÍSICO Y MEDIO AMBIENTE } \\
\hline 1) Dotación de recursos & dot_rec & $\begin{array}{l}\text { Disponibilidad de agua potable, disponibilidad de agua para riego, } \\
\text { disponibilidad de suelo agrario, disponibilidad forestal, disponibilidad de } \\
\text { espacios protegidos, disponibilidad de otros recursos, disponibilidad } \\
\text { pesquera }\end{array}$ \\
\hline $\begin{array}{l}\text { 2) Situación de los } \\
\text { Recursos }\end{array}$ & sit_rec & $\begin{array}{l}\text { Calidad del agua, calidad del suelo, calidad del aire, conservación de } \\
\text { fauna y flora, calidad del litoral, grado de contaminación acústica, } \\
\text { conservación del paisaje }\end{array}$ \\
\hline $\begin{array}{l}\text { 3) Gestión y } \\
\text { aprovechamiento de los } \\
\text { recursos }\end{array}$ & gest_rec & $\begin{array}{l}\text { Uso del suelo agrario, gestión del agua potable, gestión del agua para } \\
\text { riego, explotación de fauna y flora, recursos energéticos renovables, } \\
\text { explotación de otros recursos, gestión de residuos }\end{array}$ \\
\hline $\begin{array}{l}\text { 4) Ordenación del } \\
\text { territorio }\end{array}$ & ord_rec & $\begin{array}{l}\text { Recalificación de suelos, incidencia del PGOU en los municipios, } \\
\text { dotación de suelo uso industrial, dotación de suelo uso residencial, } \\
\text { dotación de suelo uso recreativo, dotación de infraestructuras urbanas, } \\
\text { dotación de suelos uso comercial, dotación de suelo otros usos. }\end{array}$ \\
\hline \multicolumn{3}{|c|}{ ÁREA TEMÁTICA 2: POBLACIÓN Y SOCIEDAD } \\
\hline 5) Identidad comarcal & ident & $\begin{array}{l}\text { Iniciativas empresariales locales, grado de aceptación de innovaciones y } \\
\text { cambios, participación ciudadana, conocimiento de las tradiciones } \\
\text { locales, grado de pertenencia y arraigo, argumento de la comarca, } \\
\text { atractivo de la comarca, patrimonio etnográfico }\end{array}$ \\
\hline 6) Capital Social & cap_soc & $\begin{array}{l}\text { Grado de asociacionismo, confianza en las instituciones, capacidad de } \\
\text { movilización e influencia, grado de cooperación, grado de solidaridad }\end{array}$ \\
\hline 7) Capital humano & cap_hum & $\begin{array}{l}\text { Nivel formativo de la población, dotación de profesionales, grado de } \\
\text { civismo, existencia de liderazgos, analfabetismo, analfabetismo digital }\end{array}$ \\
\hline 8) Conciliación social & conc_soc & $\begin{array}{l}\text { Integración de la inmigración, población dependiente, exclusión social, } \\
\text { accesibilidad para las personas de capacidad diversa }\end{array}$ \\
\hline
\end{tabular}

\footnotetext{
${ }^{7}$ Un constructo es un "concepto que el investigador puede definir en términos conceptuales pero que no puede ser directamente medido (...) o medido sin error" (Hair et al., 1999: 770)
} 
Tabla 1 (continuación)

Relación de áreas temáticas, macrovariables y variables

\begin{tabular}{|c|c|c|}
\hline Macrovariable & Abreviatura & Variables \\
\hline 9) Cultura y Patrimonio & cult_patri & $\begin{array}{l}\text { Dotación de infraestructuras culturales, dotación de patrimonio cultural, } \\
\text { celebración de actos culturales, apoyo público a la cultura, explotación } \\
\text { del patrimonio cultural, rutas culturales }\end{array}$ \\
\hline 10) Ocio y deporte & ocio & $\begin{array}{l}\text { Dotación de infraestructuras deportivas y de ocio, celebración de actos } \\
\text { deportivos y de ocio, apoyo público al deporte y al ocio, explotación de } \\
\text { las instalaciones deportivas y de ocio }\end{array}$ \\
\hline $\begin{array}{l}\text { 11) Formas de } \\
\text { asentamiento de la } \\
\text { Población }\end{array}$ & asent_pobl & $\begin{array}{l}\text { Población residente y temporal, grado de dependencia al municipio } \\
\text { principal, grado de identificación de diseminados con núcleo matriz }\end{array}$ \\
\hline \multicolumn{3}{|c|}{ ÁREA TEMÁTICA 3: INFRAESTRUCTURAS Y TRANSPORTE } \\
\hline $\begin{array}{l}\text { 12) Accesibilidad, } \\
\text { mantenimiento y } \\
\text { movilidad }\end{array}$ & accesib & Dotaciones viarias, servicios públicos de transporte \\
\hline $\begin{array}{l}\text { 13) Servicios básicos a } \\
\text { la población }\end{array}$ & serv_bas & $\begin{array}{l}\text { Plazas escolares primaria, plazas escolares secundaria, centro de salud, } \\
\text { dotación hospitalaria, dotación de bibliotecas y ludotecas, dotación de } \\
\text { servicios municipales básicos, dotación de servicios de guarderías, } \\
\text { dotación de plazas de educación infantil, dotación de servicios para la } \\
\text { tercera edad, dotación de servicios para atención social, dotación de } \\
\text { zonas verdes y de esparcimiento, dotación de centros de información } \\
\text { ciudadana, oferta de formación no reglada }\end{array}$ \\
\hline 14) TIC & tic & $\begin{array}{l}\text { Grado de acceso público a internet, grado de acceso privado a internet, } \\
\text { informatización de servicios públicos, informatización de las empresas, } \\
\text { acceso a las televisiones analógicas y a las digitales, acceso a la } \\
\text { telefonía móvil y fija }\end{array}$ \\
\hline $\begin{array}{l}\text { 15) Redes de } \\
\text { abastecimiento básico }\end{array}$ & abast & $\begin{array}{l}\text { Dotación de infraestructura eléctrica, dotación de infraestructura } \\
\text { gasística, dotación de infraestructura hidráulica, dotación de } \\
\text { infraestructura de gasolineras, grado de implantación de energías } \\
\text { renovables }\end{array}$ \\
\hline \multicolumn{3}{|c|}{ ÁREA TEMÁTICA 4: ECONOMÍA } \\
\hline 16) Agricultura & agric & $\begin{array}{l}\text { Tamaño de las explotaciones agrícolas, grado de abandono de la } \\
\text { actividad agrícola, diversidad de cultivos, grado de innovación agrícola, } \\
\text { acceso a regadío, grado de sostenibilidad de las explotaciones agrícolas }\end{array}$ \\
\hline 17) Pesca & pesc & $\begin{array}{l}\text { Situación de la flota pesquera, acceso a pesca, grado de abandono de la } \\
\text { actividad pesquera, grado de innovación pesquera, grado de ocupación } \\
\text { de la población pesquera }\end{array}$ \\
\hline 18) Ganadería & ganad & $\begin{array}{l}\text { Tamaño de las explotaciones ganaderas, grado de abandono de la } \\
\text { actividad ganadera, diversidad de ganados, grado de innovación } \\
\text { ganadera, grado de sostenibilidad de las explotaciones ganaderas }\end{array}$ \\
\hline $\begin{array}{l}\text { 19) Industria } \\
\text { Agroalimentaria }\end{array}$ & ind_agroal & $\begin{array}{l}\text { Existencia de agroindustria vinculada, grado de innovación } \\
\text { agroindustrial, grado de inversión agroindustria, riesgo de deslocalización } \\
\text { agroindustrial, competitividad industria agroalimentaria }\end{array}$ \\
\hline 20) Otras Industrias & otr_ind & $\begin{array}{l}\text { Grado de industrialización, diversidad empresarial, grado de innovación } \\
\text { industrial, grado de inversión industrial, riesgo de deslocalización, } \\
\text { artesanía, industria auxiliar a la agricultura }\end{array}$ \\
\hline 21) Construcción & constr & Crecimiento del número de viviendas, obras públicas \\
\hline $\begin{array}{l}\text { 22) Servicios y } \\
\text { comercio }\end{array}$ & servic_com & $\begin{array}{l}\text { Dotación de empresas de servicios no turísticos, grado de innovación en } \\
\text { servicios, comercio }\end{array}$ \\
\hline
\end{tabular}


Tabla 1 (continuación)

Relación de áreas temáticas, macrovariables y variables

\begin{tabular}{|c|c|c|}
\hline Macrovariable & Abreviatura & Variables \\
\hline 23) Actividad turística & turism & $\begin{array}{l}\text { Dotación de infraestructuras turísticas, grado de innovación turística, } \\
\text { grado de diversificación turística }\end{array}$ \\
\hline $\begin{array}{l}\text { 24) Renta y su } \\
\text { distribución }\end{array}$ & renta_dist & $\begin{array}{l}\text { Renta media de las familias, proporción de personas acogidas al } \\
\text { subsidio agrario, proporción de personas perceptoras de pensiones, } \\
\text { grado de diversidad de fuentes de ingreso, proporción de renta aportada } \\
\text { por cada sector productivo }\end{array}$ \\
\hline $\begin{array}{l}\text { 25) Redes y } \\
\text { cooperación } \\
\text { empresarial }\end{array}$ & coop_emp & $\begin{array}{l}\text { Grado de interdependencia vertical, grado de interdependencia } \\
\text { horizontal, asociacionismo empresarial, canales de distribución apoyo } \\
\text { institucional }\end{array}$ \\
\hline 26) Mercado de trabajo & mdo_tbjo & $\begin{array}{l}\text { Oferta de mano de obra, demanda de mano de obra, movilidad } \\
\text { geográfica, grado de temporalidad, intermediación laboral }\end{array}$ \\
\hline $\begin{array}{l}\text { 27) Fuentes de } \\
\text { Financiación }\end{array}$ & ftes_finan & $\begin{array}{l}\text { Dotación de entidades financieras, grado de implicación de las entidades } \\
\text { financieras, grado de endeudamiento de la población, atracción de } \\
\text { inversores }\end{array}$ \\
\hline \multicolumn{3}{|c|}{ ÁREA TEMÁTICA 5: ORGANIZACIÓN ADMINISTRATIVA } \\
\hline 28) Competencias & compet & $\begin{array}{l}\text { Cooperación entre instituciones públicas, impacto de la administración } \\
\text { central, impacto de la administración autonómica, impacto de la } \\
\text { administración provincial, impacto de la administración local, impacto del } \\
\text { grupo de acción local, organización administrativa a nivel infraprovincial }\end{array}$ \\
\hline $\begin{array}{l}\text { 29) Presencia y } \\
\text { Recursos }\end{array}$ & pres_recur & Disponibilidad y accesibilidad, utilidad y eficacia, suficiencia de recursos \\
\hline \multicolumn{3}{|c|}{ ÁREA TEMÁTICA 6: ENTORNO } \\
\hline 30) Marco legal & legal & $\begin{array}{l}\text { Impacto de la reforma de la PAC, impacto de la legislación de carácter } \\
\text { social, impacto de la legislación de carácter económico, impacto de la } \\
\text { legislación medioambiental, impacto de incentivos y ayudas, ordenación } \\
\text { del territorio }\end{array}$ \\
\hline $\begin{array}{l}\text { 31) Situación } \\
\text { macroeconómica }\end{array}$ & macroec & $\begin{array}{l}\text { Impacto de la evolución de los tipos de interés, impacto de los precios y } \\
\text { su evolución, impacto del desempleo, energía (precio y disponibilidad), } \\
\text { impacto del crecimiento económico }\end{array}$ \\
\hline $\begin{array}{l}\text { 32) Marco socio-cultural } \\
\text { y demográfico }\end{array}$ & soc_demog & $\begin{array}{l}\text { Impacto de la evolución de la población, impacto del envejecimiento de la } \\
\text { población, impacto de la inmigración, impacto del abandono de zonas } \\
\text { rurales, impacto otras tendencias sociales y culturales }\end{array}$ \\
\hline 33) Marco tecnológico & tecnol & $\begin{array}{l}\text { Impacto del uso de internet, impacto de la innovación empresarial, } \\
\text { impacto de la telefonía, impacto de la adopción de la administración } \\
\text { pública de las TICs, impacto de las energías renovables }\end{array}$ \\
\hline
\end{tabular}

Fuente: Elaboración propia.

Las macrovariables contempladas fueron tanto de carácter interno como externo y, todas ellas, conforman el territorio que se pretende analizar. Además, se consideró que fuera posible obtener información estadística publicada a través de los organismos oficiales, definiendo para ello los denominados indicadores. La lista de macrovariables no debería superar en ningún caso el número de setenta (Godet, 1993) y cada una de ellas debería estar claramente definida y caracterizada, incluyendo la tendencia de evolución en el pasado y previsible para el futuro. En total se consideraron 33 macrovariables. A su vez, 
cada macrovariable contenía un grupo de variables, de forma que el conjunto total de variables utilizado fue de 177 .

\subsubsection{La descripción de relaciones entre las macrovariables (fase 2)}

El objeto fundamental de esta fase fue el relleno de una matriz de doble entrada. Cada elemento $\left(a_{i j}\right)$ en esta matriz representa el valor de las influencias ejercidas por las variables en filas (i) sobre las variables en las columnas (j). Este es un paso clave de la técnica. Se necesita una reflexión profunda de la naturaleza de las relaciones entre elementos (Figura 2), para identificar con claridad si las influencias ejercidas por $i$ sobre $j$ son directas (a), son debidas a una tercera variable $k$ que afecta a ambas (b) o incluso si esa relación está mediatizada por el efecto de la tercera variable $m(\mathrm{c})$.

Figura 2

Tipos de influencias directas entre dos elementos

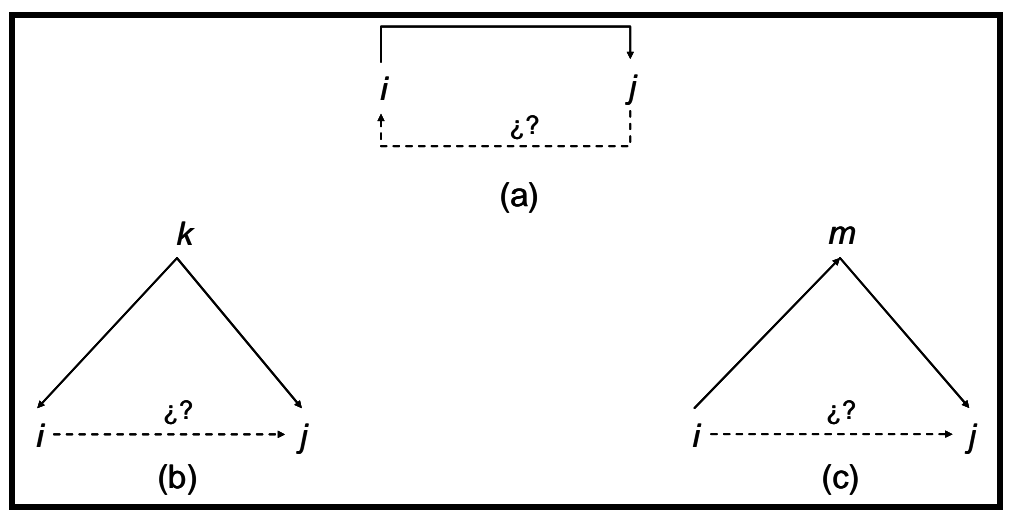

Fuente: Godet (2001: 83).

Cada elemento de la matriz $\left(a_{i j}\right)$ puede oscilar entre valores 0 y 3 (0 significa que no hay influencia directa entre dos variables, 1 se asigna a una influencia directa débil, 2 para una intensidad media y 3 para una intensidad fuerte). También se puede asignar un valor $P$ (potencial) a aquellas influencias directas que pueden ser relevantes en el futuro en el caso de que ciertas circunstancias se modifiquen. Esta herramienta no considera el signo de las influencias.

El relleno de la matriz de influencias se realizó mediante grupos de trabajo organizados en talleres de prospectiva en los que participaban los actores locales del territorio. 


\subsubsection{La identificación del papel de las macrovariables (fase 3)}

Para la clasificación de las macrovariables en el sistema se aplicó el MICMAC, un programa informático que aplica las propiedades de las matrices Booleanas para ordenar las variables conforme al número y la intensidad de las influencias que parten (influencia por motricidad) o son recibidas (influencia por dependencia) por cada variable (Godet, 1993; Godet y Bourse, 1989).

Las influencias directas entre dos variables $i$ y $j$ se consideran relaciones de orden 1, en tanto que ningún elemento media la influencia entre ambos elementos $(i \rightarrow j)$. Estas relaciones se cuantifican en la Matriz de Influencias Directas (MID), el resultado de la fase 2. Para incorporar las influencias indirectas (p.ej., $i \rightarrow k \rightarrow j$, relación de orden 2 entre $i$ y $j$ ), la sucesiva elevación de la MID a la potencia 2, 3, $4 \ldots n$ lleva a clasificar los elementos de la matriz conforme a la suma agregada de influencias ejercidas o recibidas en sucesivas iteraciones. A partir de una determinada potencia, cualquier iteración adicional no modifica la jerarquía resultante, la cual se mantiene estable y da lugar a la Matriz de Influencias Indirectas (MII).

MICMAC puede representar los elementos de un sistema en un Plano de Motricidad-Dependencia, a partir de los pares de valores asociados a cada elemento, por motricidad y por dependencia. La clasificación de las macrovariables en el Plano de Motricidad-Dependencia (Plano MD) se plantea en base a dos lógicas: una lógica de entrada-salida (Figura 3) y una lógica estratégica (Figura 4).

\section{Figura 3}

Tipificación de elementos en el plano de motricidad-dependencia según la lógica de entrada-salida

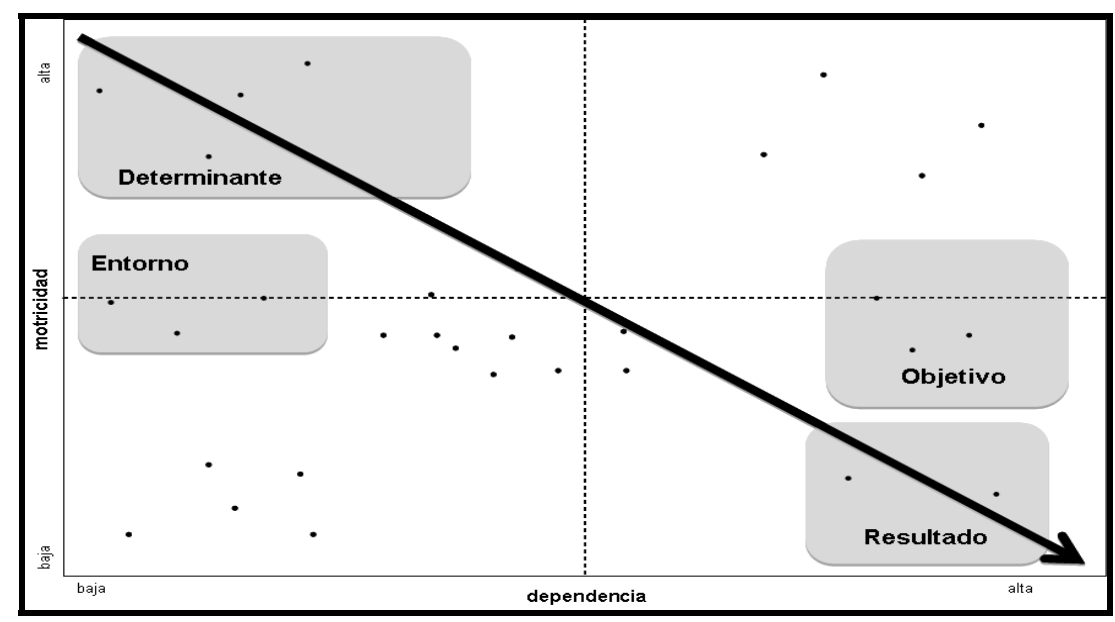

Fuente: elaboración propia, a partir de Astigarraga (blog). 
Figura 4

Tipificación de elementos en el plano de motricidad-dependencia según la lógica estratégica

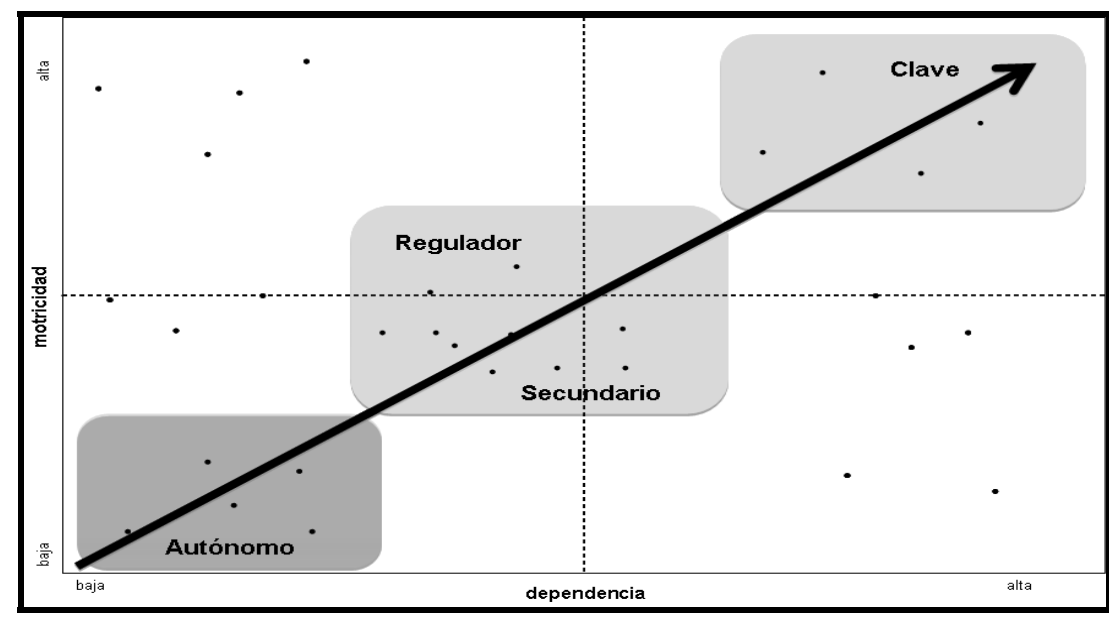

Fuente: elaboración propia, a partir de Astigarraga (blog).

La lógica de entrada-salida atraviesa el plano MD siguiendo una diagonal desde los elementos con una alta motricidad y baja dependencia hasta el extremo opuesto donde se sitúan elementos de baja motricidad y alta dependencia. Los primeros pueden interpretarse como elementos que actúan a modo de fuerzas condicionantes, con una influencia consolidada y que viene de tiempo atrás. Los elementos con una alta dependencia orientan hacia dónde se dirige el sistema, a modo de metas, aspiraciones o productos de la dinámica del sistema. Desde esta lógica distinguimos cuatro tipos de macrovariables:

- Determinantes: aquéllas que determinan el funcionamiento del sistema territorial (se considera que han tenido o están teniendo una alta incidencia en sus dinámicas) y sobre las que se piensa que otros elementos del sistema han incidido o están incidiendo poco.

- Entorno: las que se consideran que han tenido o están teniendo una incidencia media en las dinámicas territoriales y sobre las que otros elementos del sistema han incidido o están incidiendo poco.

- Objetivo: macrovariables consideradas aspiraciones manifiestas o metas del sistema territorial (se piensa que el resto de elementos ha tenido o tiene una incidencia alta en ellas, si bien su influencia en las dinámicas territoriales es media).

- Resultado: las consideradas indicadores descriptivos de la evolución del sistema territorial. Manifiestan una motricidad muy limitada (su incidencia en las dinámicas territoriales es muy baja) y una dependencia muy elevada, 
lo que las convierte en factores muy rígidos y en manifiestas debilidades del sistema.

El segundo criterio responde a una lógica estratégica y a la capacidad de provocar efectos multiplicadores, según la posición que ocupe una macrovariable en la diagonal estratégica del Plano MD (diagonal que atraviesa el plano desde el origen hasta el extremo opuesto). Esta capacidad de generar efectos multiplicadores será mayor en las macrovariables más distantes del origen del plano, y menor en las macrovariables más próximas al origen. Los elementos más cercanos al origen apenas son relevantes para el sistema, en tanto que los más alejados pueden interpretarse como retos y oportunidades de cambio, por su efecto multiplicador sirven de enlace para mediar influencias entre los elementos del sistema y de ahí que sean catalizadores de acción. Podemos distinguir cuatro tipos de macrovariables:

- Clave: aquéllas que se considera han tenido o están teniendo una alta incidencia en las dinámicas territoriales y sobre las que el resto de elementos del sistema se cree que han tenido o tienen una alta incidencia.

- Reguladoras: las que se estima han tenido o están teniendo una incidencia media en las dinámicas territoriales y sobre las que el resto de elementos del sistema se cree que han tenido o tienen una incidencia media.

- Secundarias: Macrovariables que se considera han tenido o están teniendo un impacto medio-bajo en las dinámicas territoriales y sobre los que el resto de elementos del sistema se cree que han tenido o tienen una incidencia media. Las clases secundaria y reguladora se denominan variables pelotón del sistema.

- Autónomas: aquéllas que han tenido o están teniendo un impacto bajo para generar cambios en el sistema territorial y sobre los que el resto de elementos del sistema se cree que han tenido o tienen una incidencia baja o nula.

La imagen obtenida en este plano es un producto de la interpretación que hacen los participantes de las influencias directas entre los elementos del sistema. De ahí que este plano se pueda considerar un insumo clave para entender qué restricciones, oportunidades y dinamizadores del cambio perciben los actores locales y, en consecuencia, afronta su territorio rural.

\section{RESULTADOS Y DISCUSIÓN}

\subsection{El plano de motricidad-dependencia en la comarca de Jerez}

Los resultados que se presentan corresponden a la aplicación del método del AEP a la comarca de Jerez (Cádiz). La información de la comarca fue plasmada en la Matriz de Influencias Directas (MID) después de varias sesiones de trabajo 
en las denominadas mesas comarcales de prospectiva. La MID está formada de 33 filas y 33 columnas correspondientes a las 33 macrovariables (Tabla 2).

Tabla 2

Matriz de Influencias Directas para la comarca de Jerez

\begin{tabular}{|c|c|c|c|c|c|c|c|c|c|c|c|c|c|c|c|c|c|c|c|c|c|c|c|c|c|c|c|c|c|c|c|c|c|c|}
\hline & & & & $\begin{array}{l}m \\
5 \\
\mathbf{2} \\
\mathbf{2}\end{array}$ & $\begin{array}{l}\nabla \\
\vdots \\
\Sigma \\
\Sigma\end{array}$ & $\stackrel{r}{\mathrm{i}}$ & 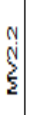 & $\begin{array}{l}m \\
\stackrel{m}{2} \\
\frac{2}{2}\end{array}$ & $\begin{array}{l}\vec{\nabla} \\
\grave{3} \\
\grave{2}\end{array}$ & $\stackrel{n}{\mathrm{n}}$ & $\begin{array}{l}0 \\
\stackrel{0}{S} \\
\frac{2}{2}\end{array}$ & & & & & & & & & & & & & 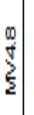 & $\frac{9}{3}$ & 0 & $\frac{1}{2}$ & $\begin{array}{l}\mathrm{N} \\
\mathrm{r} \\
\mathrm{J} \\
\frac{2}{2}\end{array}$ & 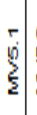 & 0 & $\frac{r}{\xi}$ & $\begin{array}{l}N \\
0 \\
\vdots \\
2\end{array}$ & $\begin{array}{l}n \\
9 \\
2 \\
2\end{array}$ & \\
\hline Dotación de recursos & M1.1 & 0 & 0 & 0 & 0 & 0 & 0 & 0 & 0 & 0 & 0 & 0 & 0 & 0 & 0 & 0 & 0 & 1 & 0 & 0 & 0 & 0 & 0 & 0 & 0 & 0 & 0 & 0 & 0 & 0 & 0 & 0 & 0 & \\
\hline Situación de los Rearsos & MN1.2 & 2 & 0 & 3 & 3 & 3 & 1 & 2 & 0 & 1 & 2 & 1 & 2 & 1 & 0 & 12 & 2 & 3 & 1 & 1 & 2 & 2 & 0 & 1 & 0 & 0 & 0 & 0 & 2 & 2 & 2 & 0 & 0 & 0 \\
\hline Gestión y sprovechamiento de los recursos & MN1.3 & 3 & 3 & 0 & 2 & 2 & 1 & 2 & 1 & 3 & 2 & 2 & 3 & 2 & 2 & 2 & 2 & 1 & 2 & 1 & 2 & 2 & 1 & 3 & 1 & 2 & 0 & 2 & 2 & 2 & 2 & 1 & 0 & \\
\hline Ordensción del territorio & MN1.4 & 2 & 2 & 1 & 0 & 2 & 1 & 1 & 0 & 1 & 0 & 3 & 2 & 0 & 1 & 2 & 2 & 0 & 1 & 2 & 1 & 2 & 1 & 2 & 0 & 1 & 0 & 0 & 3 & 2 & 3 & 1 & 1 & 0 \\
\hline Idenidod comarcal & MN21 & 3 & 2 & 1 & 2 & 0 & 3 & 3 & 2 & 3 & 1 & 2 & 2 & 2 & 1 & 23 & 3 & 0 & 1 & 1 & 0 & 1 & 1 & 1 & 2 & 2 & 2 & 0 & 0 & 2 & 3 & 0 & 2 & 0 \\
\hline Capital Social & MN22 & 1 & 1 & 2 & 1 & 3 & 0 & 3 & 2 & 1 & 1 & 1 & 1 & 3 & 1 & 2 & 2 & 0 & 2 & 2 & 0 & 0 & 0 & 1 & 2 & 1 & 2 & 0 & 1 & 2 & 1 & 0 & 2 & 0 \\
\hline Capital humano & MN23 & 1 & 1 & 1 & 1 & 3 & 3 & 0 & 1 & 2 & 2 & 1 & 1 & 3 & 1 & 0 & 1 & 0 & 1 & 1 & 0 & 0 & 0 & 1 & 1 & 1 & 1 & 0 & 0 & 2 & 1 & 0 & 2 & 0 \\
\hline Conciligaón socis & MN24 & 0 & 0 & 1 & 1 & 1 & 2 & 1 & 0 & 0 & 1 & 1 & 0 & 1 & 1 & 0 & 1 & 0 & 0 & 1 & 0 & 2 & 0 & 1 & 1 & 1 & 2 & 0 & 1 & 2 & 1 & 0 & 2 & 0 \\
\hline Cuturay Patrimonio & MN25 & 3 & 3 & 3 & 2 & 3 & 2 & 1 & 0 & 0 & 1 & 3 & 2 & 1 & 1 & 0 & 1 & 0 & 1 & 0 & 0 & 0 & 2 & 3 & 1 & 0 & 1 & 1 & 3 & 2 & 1 & 0 & 2 & \\
\hline Ocioy deporte & MN26 & 3 & 1 & 3 & 2 & 3 & 2 & 2 & 1 & 1 & 0 & 1 & 2 & 1 & 1 & 10 & 0 & 0 & 0 & 0 & 0 & 0 & 1 & 3 & 1 & 0 & 1 & 1 & 3 & 2 & 1 & 0 & 2 & \\
\hline Formas de asentamiento de la Población & MN27 & 1 & 0 & 1 & 3 & 2 & 2 & 1 & 1 & 1 & 2 & 0 & 3 & 3 & 2 & 3 & 0 & 0 & 0 & 0 & 0 & 0 & 2 & 1 & 0 & 1 & 3 & 2 & 3 & 3 & 3 & 1 & 0 & 1 \\
\hline Acossibilidgd, mantenimiento y movilidgd & MN3.1 & 2 & 1 & 2 & 3 & 1 & 0 & 0 & 0 & 1 & 0 & 2 & 0 & 2 & 0 & 0 & 2 & 0 & 1 & 1 & 3 & 2 & 0 & 1 & 0 & 1 & 0 & 0 & 3 & 1 & 2 & 1 & 0 & 1 \\
\hline Servicios básicos a la población & MN3.2 & 0 & 0 & 1 & 3 & 1 & 1 & 1 & 1 & 1 & 1 & 2 & 1 & 0 & 1 & 2 & 0 & 0 & 0 & 0 & 0 & 1 & 1 & 0 & 0 & 0 & 1 & 2 & 3 & 1 & 3 & 1 & 1 & 1 \\
\hline TIC & MN3.3 & 0 & 0 & 0 & 1 & 1 & 0 & 0 & 0 & 1 & 1 & 2 & 0 & 3 & 0 & 0 & 0 & 0 & 0 & 1 & 1 & 0 & 1 & 1 & 0 & 1 & 1 & 2 & 2 & 1 & 2 & 1 & 1 & 2 \\
\hline Redes de abas & MN3.4 & 0 & 1 & 1 & 3 & 1 & 0 & 1 & 0 & 0 & 0 & 2 & 1 & 1 & 0 & 0 & 1 & 0 & 0 & 1 & 1 & 2 & 1 & 0 & 0 & 1 & 0 & 0 & 3 & 1 & 1 & 0 & 0 & 1 \\
\hline Agrialtura & MN4.1 & 2 & 3 & 3 & 3 & 1 & 1 & 1 & 1 & 1 & 0 & 1 & 1 & 1 & 1 & 3 & 0 & 0 & 1 & 3 & 2 & 1 & 2 & 1 & 2 & 3 & 3 & 3 & 3 & 2 & 3 & 3 & 1 & 2 \\
\hline Pesca & MN4.2 & 2 & 3 & 2 & 1 & 1 & 1 & 1 & 1 & 1 & 0 & 1 & 0 & 1 & 0 & 2 & 0 & 0 & 0 & 1 & 1 & 0 & 1 & 0 & 2 & 1 & 3 & 3 & 3 & 2 & 3 & 2 & 1 & 0 \\
\hline Gangderia & MN4.3 & 2 & \begin{tabular}{|l|l}
3 \\
\end{tabular} & 3 & 3 & 1 & 1 & 1 & 1 & 1 & 0 & 1 & 1 & 1 & 1 & 3 & 0 & 0 & 0 & 3 & 2 & 0 & 2 & 1 & 2 & 2 & 3 & 3 & 3 & 1 & 3 & 3 & 1 & 2 \\
\hline Industria Agroalimentaria & MN4.4 & 2 & 0 & 3 & 3 & 1 & 1 & 1 & 0 & 0 & 0 & 1 & 3 & 1 & 2 & 3 & 3 & 1 & 3 & 0 & 0 & 1 & 2 & 0 & 2 & 0 & 2 & 2 & 3 & 2 & 3 & 2 & 0 & 3 \\
\hline Otras Industrias & MN4.5 & 0 & 0 & 2 & 3 & 1 & 1 & 1 & 0 & 0 & 0 & 2 & 3 & 1 & 2 & 32 & 2 & 1 & 1 & 2 & 0 & 2 & 1 & 1 & 2 & 1 & 2 & 2 & 3 & 2 & 3 & 2 & 0 & 3 \\
\hline Construcción & MN4.6 & 1 & 1 & 2 & 3 & 1 & 0 & 1 & 1 & 0 & 0 & 3 & 3 & 1 & 1 & 3 & 0 & 0 & 0 & 0 & 3 & 0 & 1 & 0 & 2 & 1 & 2 & 2 & 2 & 1 & 2 & 2 & 1 & 2 \\
\hline Servicios y comecio & MN4.7 & 0 & \begin{tabular}{|l|}
2 \\
\end{tabular} & 1 & 3 & 1 & 1 & 0 & 1 & 1 & 1 & 3 & 2 & 2 & 1 & 1 & 1 & 1 & 1 & 2 & 1 & 2 & 0 & 2 & 2 & 1 & 2 & 3 & 1 & 2 & 2 & 1 & \begin{tabular}{|l|}
1 \\
\end{tabular} & 1 \\
\hline Actividgo turística & MN4.8 & 3 & 3 & 3 & 2 & 1 & 1 & 1 & 1 & 3 & 2 & 3 & 3 & 1 & 3 & 2 & 2 & 1 & 2 & 0 & 0 & 2 & 2 & 0 & 2 & 2 & 2 & 2 & 2 & 2 & 2 & 2 & 2 & 2 \\
\hline Renta y su distribución & MN4.9 & 1 & 1 & 2 & 2 & 0 & 0 & 1 & 1 & 0 & 1 & 2 & 2 & 1 & 0 & 0 & 2 & 0 & 2 & 2 & 1 & 3 & 1 & 2 & 0 & 1 & 3 & 2 & 1 & 1 & 1 & 3 & 0 & 1 \\
\hline Redes $y 0$ & MN4.10 & 1 & 1 & 1 & 1 & 0 & 2 & 2 & 0 & 1 & 0 & 1 & 0 & 0 & 2 & 0 & 1 & 0 & 1 & 2 & 2 & 1 & 1 & 2 & 1 & 0 & 1 & 2 & 1 & 2 & 1 & 2 & 0 & 1 \\
\hline Mercado de trabajo & MN4.11 & 1 & 1 & 2 & 1 & 1 & 1 & 2 & 2 & 1 & 1 & 2 & 2 & 0 & 2 & 0 & 1 & 1 & 2 & 2 & 2 & 3 & 0 & 1 & 3 & 1 & 0 & 2 & 1 & 1 & 2 & 3 & 2 & 2 \\
\hline Fuentes de Financisción & MN4.12 & 0 & 1 & 1 & 1 & 0 & 0 & 0 & 0 & 1 & 0 & 2 & 1 & 0 & 0 & 0 & 1 & 0 & 0 & 0 & 1 & 1 & 0 & 0 & 2 & 1 & 1 & 0 & 1 & 1 & 1 & 3 & \begin{tabular}{|l|}
1 \\
\end{tabular} & 1 \\
\hline Competenciss & MN5.1 & 0 & 0 & 0 & 1 & 0 & 0 & 0 & 0 & 0 & 0 & 2 & 0 & 0 & 1 & 0 & 0 & 0 & 0 & 0 & 0 & 0 & 0 & 0 & 0 & 0 & 0 & 0 & 0 & 1 & 3 & 2 & 0 & 1 \\
\hline Presencia y Recurs os & M5.2 & 0 & 0 & 0 & 0 & 1 & 0 & 0 & 0 & 1 & 0 & 3 & 0 & 0 & 1 & 0 & 1 & 0 & 1 & 1 & 0 & 0 & 0 & 0 & 0 & 0 & 1 & 0 & 3 & 0 & 2 & 1 & 1 & 2 \\
\hline Marco legal & MN6.1 & 1 & 0 & 0 & 0 & 0 & 0 & 0 & 0 & 0 & 0 & 0 & 0 & 0 & 0 & 0 & 0 & 0 & 0 & 0 & 0 & 0 & 0 & 0 & 0 & 0 & 0 & 0 & 1 & 0 & 0 & 2 & 2 & 0 \\
\hline Situgción macu oeconónica & MN6.2 & 0 & 0 & 0 & 0 & 0 & 0 & 0 & 0 & 0 & 0 & 0 & 0 & 0 & 0 & 0 & 0 & 0 & 0 & 0 & 0 & 0 & 0 & 0 & 0 & 0 & 0 & 0 & 0 & 0 & 3 & 0 & 0 & 1 \\
\hline Marcos ocio-aultural y denog áfico & MN6.3 & 0 & 0 & 0 & 0 & 0 & 0 & 0 & 0 & 0 & 0 & 0 & 0 & 0 & 0 & 0 & 0 & 0 & 0 & 0 & 0 & 0 & 0 & 0 & 0 & 0 & 0 & 0 & 0 & 0 & 3 & 2 & 0 & \\
\hline Marco tecrologicos & MV6.4 & 0 & 0 & 0 & 0 & 0 & 0 & 0 & 0 & 0 & 0 & 0 & 0 & 0 & 0 & 0 & 0 & 0 & 0 & 0 & 0 & 0 & 0 & 0 & 0 & 0 & 0 & 0 & 0 & 0 & 3 & 2 & 1 & \\
\hline
\end{tabular}


El porcentaje de relleno de la matriz de influencias directas (MID) fue del $61 \%^{8}$, y se optó por no realizar ninguna transformación de la matriz original. A partir de esta matriz se obtuvo el Plano MD que se muestra en la Figura 5 mediante el programa informático MICMAC (Godet y Bourse, 1989).

Figura 5

Plano de motricidad-dependencia por influencias directas de la comarca de Jerez

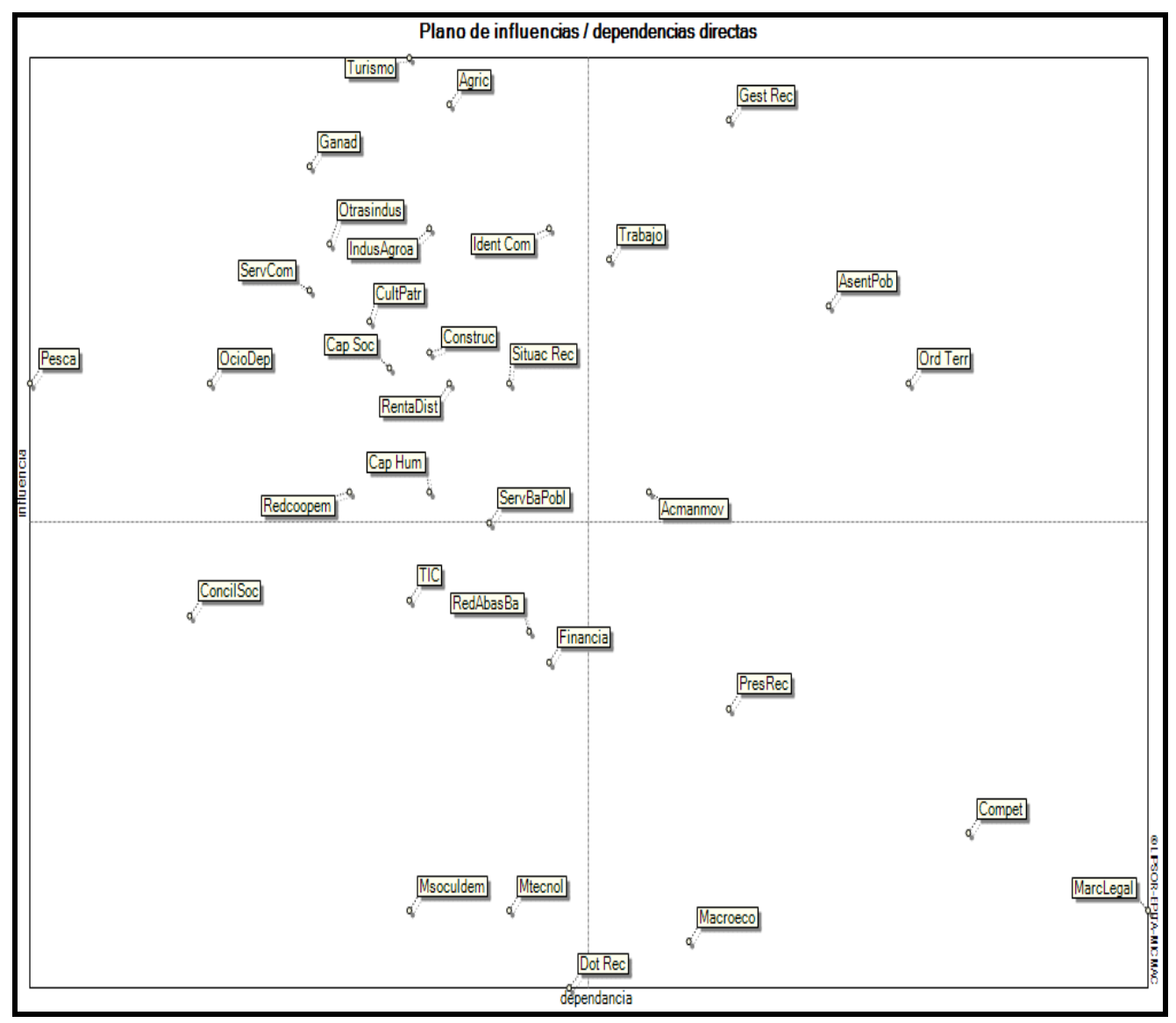

Fuente: elaboración propia, resultado del programa MICMAC (Godet y Bourse, 1989).

${ }^{8}$ La tasa de relleno de la Matriz de Influencias Directas (MID) se calcula como el porcentaje de valores distintos a cero sobre el total de celdas de la matriz MID (excluyendo los valores de la diagonal). Una tasa aceptable de relleno de la MID debería estar en torno al $25 \%$. Tasas superiores indican que se han interpretado relaciones indirectas como si fuera directas (Godet, 1993), aunque también se pueden encontrar tasas alrededor del 50\% (Astigarraga, 2006). 
Para agrupar las macrovariables según las dos lógicas indicadas (entradasalida y estratégica) se siguieron los siguientes pasos:

a) Calcular el valor estratégico de las macrovariables: Los valores estratégicos se calcularon a partir de la suma de las proporciones por Influencia directa y por Dependencia directa. Estos valores de proporciones son provistos por el programa MICMAC.

b) Discriminar las macrovariables pelotón: Para discriminar las macrovariables pelotón (reguladoras y secundarias) se obtuvo el promedio de los valores estratégicos calculados en el paso anterior. Las macrovariables pelotón serán aquéllas cuyo valor estratégico se encuentra en el intervalo definido por los siguientes límites ${ }^{9}$ :

- como límite superior, el 115\% del promedio de los valores estratégicos;

- como límite inferior, el 85\% del promedio de los valores estratégicos.

c) Discriminar las macrovariables reguladoras y las macrovariables secundarias: Al considerar los resultados de la MID, se utilizaron las proporciones por influencia directa:

- el valor promedio se calculó a partir de las proporciones por influencia directa del total de macrovariables (sean o no pelotón), y

- los valores a comparar (de las macrovariables pelotón) fueron las proporciones por influencia directa.

A continuación se muestra el resultado de agrupar las macrovariables siguiendo la lógica de entrada-salida (Figura 6) y la lógica estratégica (Figura 7).

\footnotetext{
${ }^{9}$ La tasa del $15 \%$ se ha utilizado a modo de valor heurístico.
} 
Figura 6

Tipificación de macrovariables en la comarca de Jerez según lógica de entrada-salida

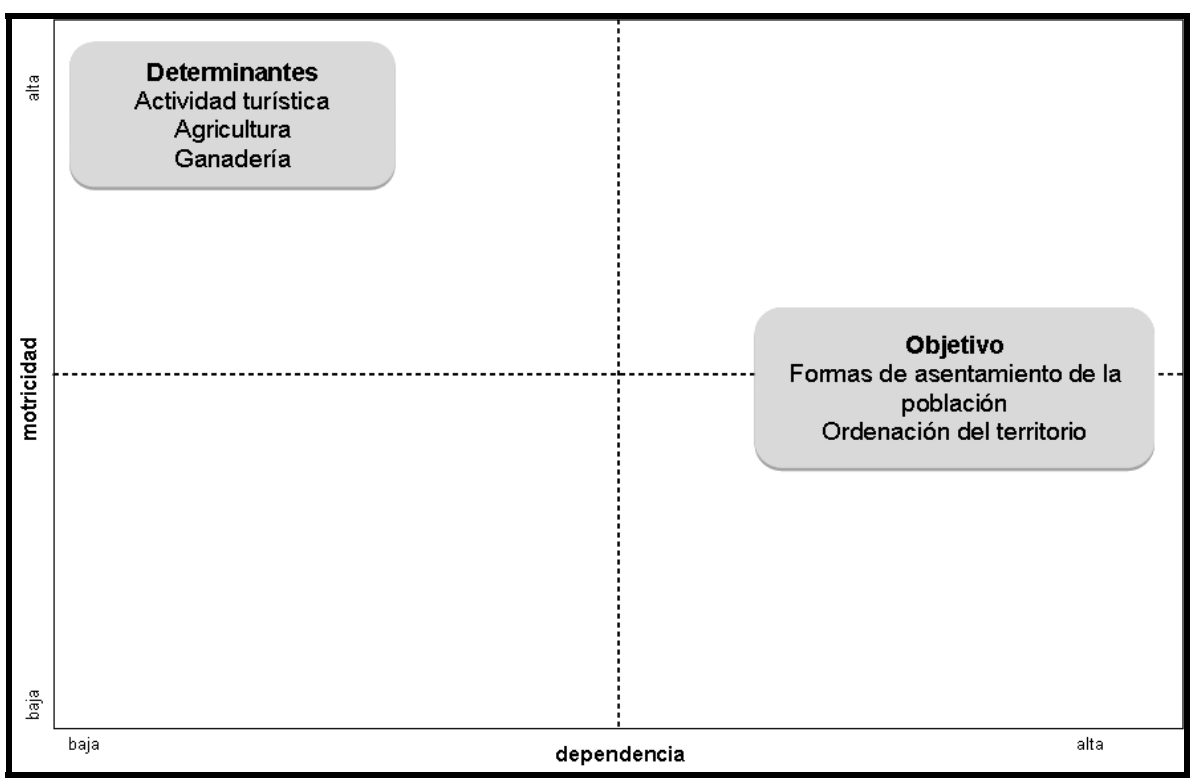

Fuente: elaboración propia.

Figura 7

Tipificación de macrovariables en la comarca de Jerez según lógica estratégica

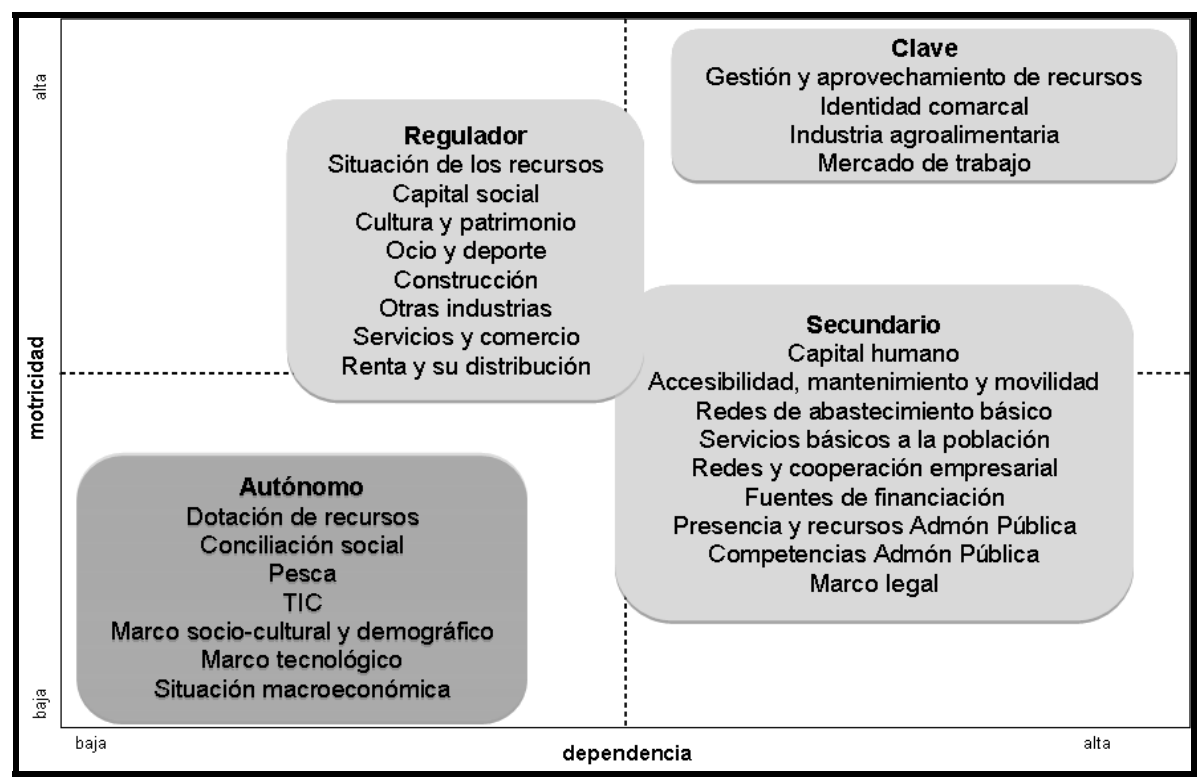

Fuente: elaboración propia. 


\subsection{El papel de las macrovariables en el sistema comarcal de Jerez}

El análisis de los resultados obtenidos requiere de un estudio detallado teniendo en cuenta las lógicas establecidas por la metodología del análisis estructural de prospectiva: lógica de entrada-salida y lógica estratégica.

\subsubsection{Determinantes y aspiraciones de Jerez (lógica de entrada-salida)}

Sobre la base de los resultados obtenidos (Figura 6), las dinámicas territoriales en la comarca de Jerez están condicionadas por tres macrovariables del área Economía: Agricultura, Ganadería y Actividad turística. Las actividades asociadas a las dos primeras determinan los procesos territoriales en la comarca derivando su actividad hacia la industria alimentaria, principalmente en el municipio de Jerez. En este sentido, cabe considerar que el efecto positivo de la multifuncionalidad en la agricultura podría justificar socialmente el apoyo público (Kallas y Gómez-Limón, 2007) sólo en la medida en que éste genere, como es el caso, externalidades positivas y contribuya de esta manera favorable al bienestar social de una comarca. En cuanto al turismo, tiene una importancia fundamental en esta comarca aportando una parte muy elevada de la producción de forma directa e indirecta (téngase en cuenta que además del municipio de Jerez, la comarca cuenta con los municipios del Puerto de Santa María y de Puerto Real, en los que esta actividad económica es vital para su subsistencia). Estas macrovariables se perciben como factores externos, sobre los que sí cabe alguna incidencia desde el territorio ya que se sitúan relativamente próximos al valor promedio de la dependencia.

Como objetivos o aspiraciones del territorio, se identifican las siguientes variables: Formas de asentamiento de la población y Ordenación del territorio.

Conforme a las variables que integran estas macrovariables, esta posición sugiere que la distribución de los núcleos de población acarrea dificultades para la prestación de los servicios básicos a la población. De hecho Jerez es uno de los ayuntamientos de mayor extensión de España con más de una veintena de entidades -siete pedanías y dieciséis barriadas rurales- alejadas entre cuatro y veinte kilómetros del centro urbano y, al mismo tiempo, las comunicaciones con los otros dos mayores núcleos de población de la comarca (El Puerto de Santa María y Puerto Real), requieren del impulso de nuevas redes viarias y de la mejora de las existentes, así como de nuevos proyectos urbanísticos ante las necesidades crecientes de suelo para edificación.

\subsubsection{Elementos estratégicos de Jerez (lógica estratégica)}

Las macrovariables estratégicas conjugan niveles relativamente parejos de motricidad y de dependencia, lo que indica capacidad para generar efectos multiplicadores (mayor en las macrovariables clave y menor en las autónomas). 
Según las percepciones del GAL, las macrovariables con un mayor efecto multiplicador (clave) en la comarca de Jerez fueron: Mercado de trabajo, Gestión y aprovechamiento de los recursos, Industria agroalimentaria e Identidad local, situadas en el extremo de la diagonal del plano (Figura 7).

Los procesos territoriales están fuertemente sesgados por el aprovechamiento del medio natural y la industria agroalimentaria. En un territorio en el que el sector agrario es percibido como macrovariable determinante, es lógico que el motor económico sea una actividad agrícola modernizada, muy condicionada por la situación del medio natural, pero con un componente intenso de innovación tecnológica (si fuera una necesidad o aspiración del territorio, estaría situada como objetivo). No obstante, la gran influencia del turismo puede provocar que el objetivo de innovación tecnológica no sea percibido como tal.

Encontrar al mercado del trabajo dentro de este grupo se debe además de a la innovación tecnológica, a la aplicación de la PAC que afectó negativamente al empleo generado por este sector con un sensible aumento del paro y a la temporalidad del empleo en los sectores agrario, turístico y construcción.

Una identidad comarcal clave apunta a un territorio con un fuerte arraigo y sentido de pertenencia de la población. Conforme a otras variables consideradas, también sugiere un grado considerable de innovación e iniciativa empresarial local, si bien esto debería contrastarse con las supuestas dificultades para asumir la reestructuración del mercado laboral.

En cualquier caso, esta percepción fuerte de su patrimonio etnográfico y de los valores comunes condiciona cualquier dinámica territorial. Dada la posición de la macrovariable formas de asentamiento de la población y el efecto centrípeto ejercido por los núcleos principales, cabría también considerar que identidad comarcal viene muy marcada por los municipio principales del territorio que anulan otras identidades rurales de los pequeños asentamientos.

Con un efecto multiplicador algo menor, pero siempre relevante para los procesos territoriales (macrovariables reguladoras) se encuentran: Otras industrias, Servicios y comercio, Construcción, Renta y su distribución, Cultura y patrimonio, Ocio y deporte, Capital social, y Situación de los recursos.

La posición de otras actividades económicas en una categoría con una relevancia estratégica considerable (aunque no la mayor) se interpreta como una diversificación de la estructura económica que está ejerciendo un impacto notable en los procesos de cambio territoriales. La situación de Renta y su distribución podría estar referida -según las variables integrantes- a la relevancia de las ayudas públicas (en forma de subsidios al desempleo agrario, ayudas de la $\mathrm{PAC}$, gastos públicos, etc.) u otro tipo de prestaciones para la población comarcal (lo que podría vincularse a la hipótesis de la reestructuración del mercado laboral), a la precariedad en los ingresos 
familiares, a una distribución desigual o a un aumento de las fuentes de renta (conforme a la suposición de una estructura económica diversificada). Con independencia de las posibles interpretaciones, sí se trata de un aspecto muy significativo para las dinámicas comarcales.

Otras macrovariables con impacto relevante en las dinámicas comarcales, y que aparentemente podrían ser calificadas como factores sin importancia, están vinculadas con la existencia de infraestructuras y el aprovechamiento del patrimonio cultural y con la explotación de actividades de ocio y deportivas (que probablemente ofrezcan otras evidencias de diversificación de la estructura económica comarcal) y con el capital social territorial siendo ésta una valoración extensible al conjunto de la comarca.

La macrovariable situación de los recursos debería interpretarse considerando que gestión y aprovechamiento de los recursos es clave y que dotación de recursos es autónoma. Muy probablemente, se perciba que el aprovechamiento que desde la comarca se consigue hacer de los recursos naturales (de cualquier tipo) está mucho más condicionado por el estado de conservación y la calidad de estos activos que propiamente por la dotación existente (al ser autónomo, quizás se considere que ésta no sea una limitante o debilidad).

Con un efecto multiplicador menor, y con menos relevancia para las dinámicas territoriales se encuentran las macrovariables secundarias: Redes y cooperación empresarial, Fuentes de financiación, Accesibilidad, mantenimiento y movilidad, Servicios básicos a la población, Redes de abastecimiento básico, Presencia y recursos, Competencias, Capital humano y Marco legal.

Obsérvese que en la comarca de Jerez las macrovariables reguladoras abarcan grados de motricidad más diversos (Figura 7): desde una motricidad media (redes y cooperación empresarial, capital humano, servicios básicos a la población y accesibilidad) hasta una motricidad muy baja (competencia y marco legal).

Dada su naturaleza, las macrovariables de motricidad media sugieren pilares estructurales del sistema territorial. Tienen cierta importancia para la comarca, pues su posición indica una capacidad notable para generar efectos multiplicadores en otros procesos territoriales (redes y cooperación empresarial; capital humano; servicios básicos a la población; accesibilidad, mantenimiento y movilidad). En la medida que se incida en ellas, se pueden provocar impactos en otras macrovariables reguladoras y clave. De hecho estas cuatro macrovariables son muy necesarias para el desarrollo de del territorio ya que con ellas los sectores productivos (agrario e industrias afines y turístico) podrían mejorar el valor añadido y su productividad. En las discusiones mantenidas en las mesas de debate se hizo un gran hincapié en la falta de 
cooperación entre las industrias y en las dificultades que ésta creaba para una buena distribución de los productos y servicios, incluyendo también las dificultades en el acceso y movilidad por las carencias en las infraestructuras.

Una interpretación similar se podría hacer para las macrovariables Redes de abastecimiento básico y Fuentes de financiación. Su capacidad para generar efectos multiplicadores se considera menor, ya que para la segunda variable los agentes consideraron que ésta viene dada desde fuera y sobre ella poco se puede hacer, en cambio las redes de abastecimiento básico se consideraron esenciales para la población distinguiendo en las mesas de debate entre aquélla que se encuentra satisfecha en los grandes núcleos poblacionales, y la que se encuentra en las pequeñas poblaciones en las que existe ausencia o deficiencia de determinados servicios básicos.

Quizás, sí cabría observar más detenidamente las macrovariable del área Organización Administrativa (Presencia y recursos y Competencias) ${ }^{10}$. Apuntan a un territorio en el que se percibe escasamente la acción pública y por lo tanto no se estima que ésta la condicione, ambas macrovariables tienen un impacto bajo en las dinámicas territoriales con baja motricidad, si bien reciben una influencia importante (ver en Figura 7 los valores medio y alto de dependencia por la posición de estas dos macrovariables). Esta última interpretación indica una demanda importante de acción pública en el territorio, motivada por las necesidades de la generación de puestos de trabajo, la revitalización económica de la comarca y la ordenación del territorio, además de las deficiencias y carencias de los servicios básicos a la población anteriormente mencionados. En este sentido estas macrovariables fueron tratadas en diversas mesas de debate relacionadas con el área temática de Economía.

Por último, macrovariables con un valor estratégico menor (autónomas), se encuentran: Pesca, Conciliación social, Tecnologías de la información y la comunicación, Marco tecnológico, Situación macroeconómica, Dotación de recursos y Marco socio-cultural demográfico. También habría que distinguir al menos tres grandes grupos de macrovariables, según el grado de motricidad que manifiestan (Figura 7).

La macrovariable Pesca aparentemente podría ser considerada un elemento de entorno (véase las pautas para la interpretación). Ya sea como autónoma o como entorno, es una actividad con un impacto notable, aunque se percibe inaccesible a la influencia de la comarca (sea por la escasa capacidad de incidir en la política de cuotas, porque la flota pesquera responde a inversores externos,

\footnotetext{
${ }^{10}$ La clasificación como 'secundarias' resulta de aplicar el procedimiento algebraico expuesto. No obstante, si observamos el Plano MD de Jerez (Figura 5), veremos que estas dos macrovariables se posicionan en el extremo inferior derecho, correspondiente a los 'elementos resultado' y que haría más coherente nuestra interpretación. Hecha esta aclaración, mantenemos los resultados obtenidos con el procedimiento algebraico.
} 
por el agotamiento de los caladeros locales o la subordinación a caladeros fuera del área de influencia de la comarca).

Con una motricidad media (pero siempre autónomas), las macrovariables Conciliación social y TIC sugieren, en el caso de la primera, una escasa atención e incidencia (dependencia) en la población en situación de precariedad y/o de exclusión (inmigrantes, población dependiente, bolsas de marginalidad) aunque se trate de una cuestión con cierta relevancia para la comarca. En el caso de las TIC, éstas también impactan en las dinámicas territoriales, aunque la incidencia en ellas (inversión pública y privada, informatización de servicios) es más significativa.

Por último, se sitúan macrovariables que apenas tienen incidencia en las dinámicas de la comarca. Tres pertenecen al área Entorno: Marco socio-cultural y demográfico (envejecimiento, pérdida de población joven, inmigración), la Situación macroeconómica ${ }^{11}$ y el Marco tecnológico; y la cuarta pertenece al área Medio físico y Medio ambiente -Dotación de recursos-, para cuya interpretación se remite al lector al subapartado dedicado a las macrovariables reguladoras. Únicamente en relación a la situación macroecómica puede decirse que tiene un nivel de dependencia medio lo que denota que la evolución de los precios, el tipo de interés y en general el crecimiento de la economía del país es muy importante para la evolución del territorio en su conjunto aunque éste tenga pocas posiblidades de influir sobre esta macrovariable.

\section{CONCLUSIONES}

La última reglamentación comunitaria sobre desarrollo rural reafirma el carácter territorial con el que la Unión Europea dota a sus políticas para la zonas rurales. La Iniciativa LEADER y los sucesivos programas nacionales y regionales puestos en marcha han hecho de la red de grupos y organizaciones territoriales una seña fundamental de ese carácter territorial de la política rural. Este carácter confiere a los grupos y redes territoriales un papel medular en el diseño e implementación de programas locales que contribuyen a objetivos y líneas estratégicas más amplios.

Además de sus implicaciones para gestionar una política pública, este enfoque territorial de la política rural también ofrece nuevas vías para mejorar nuestra comprensión de los procesos de desarrollo que tienen lugar en una zona rural. En la medida que los GAL emergen e interactúan con el tejido social, económico y político de estas zonas, se convierten en protagonistas singulares del cambio rural, a la par que en interlocutores únicos para quienes se aproximan a estos territorios desde el exterior. Mejorar la comprensión de los

\footnotetext{
${ }^{11}$ La estrategia se llevó a cabo a lo largo del año 2007, año en el que la crisis económica no había comenzado a ser atisbada por los ciudadanos.
} 
incentivos y obstáculos a los procesos de cambio en estas zonas, involucrando a los propios actores rurales en dicha deliberación, es un condición indispensable para hacer que las políticas públicas rurales sean más eficaces.

El proyecto NERA es un estudio pionero en la Unión Europea que muestra un camino posible para el diseño de propuestas estratégicas de manera coordinada en territorios concretos y en una región, como la comunidad autónoma andaluza, con un marcado carácter rural. Este proyecto es un modelo de participación de la sociedad rural en su conjunto en el proceso de debate del futuro del mundo rural andaluz. Así, con el proyecto NERA se ha implementado una estrategia que se integra plenamente en la propuesta política de desarrollo sostenible para la región, impulsada por el gobierno andaluz.

Con el objetivo de indagar en el comportamiento y la influencia de las principales variables socioeconómicas que definen el mundo rural andaluz, en el diseño de la NERA se aplicó el método del AEP. En este artículo se ha expuesto la aplicación realizada en la comarca de Jerez de este método y los resultados obtenidos.

El método del AEP ofrece algunos rasgos de especial relevancia para su aplicación al estudio de las zonas rurales. Entre otros, destacaríamos que no es un método costoso en términos monetarios ni demanda mucho tiempo para su realización. Al basarse en información cualitativa es idóneo para zonas con escasa información estadística o de poca calidad. En términos conceptuales, permite realizar un análisis funcional y dinámico de los elementos del sistema territorial. Al conjugar las características de motricidad y dependencia, ayuda a identificar la función que desempeña cada elemento en los procesos de cambio del territorio. En vez de considerar relaciones de causa-efecto, este análisis dinámico se sustenta en las múltiples relaciones de influencia no lineales entre los elementos del sistema. De ahí que ofrezca una aproximación más real al comportamiento de los elementos del territorio.

La elaboración de un consenso previo sobre la lista y la definición de las macrovariables con los participantes es un requisito básico para que la deliberación sea eficaz y se haga con eficiencia. El rellenado de la matriz de influencias directas es un momento clave. A la par que permite abordar en profundidad las relaciones de influencia entre los factores que conforman el sistema territorial, esta matriz estructura la discusión y reduce el riesgo de dispersión en las deliberaciones, contribuyendo significativamente a un debate más eficaz. La efectividad de este método se sustenta en la implicación y el compromiso sostenido de los actores locales clave durante las deliberaciones para rellenar la matriz. El logro de cualquier cambio deseado en las zonas rurales no se apoya tan solo en el conocimiento y la experiencia locales, sino sobre todo en su implicación en el proceso de cambio y su capacidad para dirigirlo. 
La aplicación del programa informático MICMAC para procesar la matriz resultante de las mesas de discusión ayuda a reforzar la confianza de los participantes en los resultados obtenidos, reduciendo cualquier sospecha sobre eventuales prioridades que respondieran a intereses particulares en lugar de ser resultado de la deliberación y el acuerdo colectivos. Su aplicación facilita la labor de los GAL en el diseño de las prioridades estratégicas, al tener un claro conocimiento de qué función desempeñaban las variables consideradas.

$\mathrm{Su}$ aplicación a la comarca de Jerez permite concluir que es un método sencillo y eficaz para analizar y deliberar de forma estratégica sobre lo que acontece en un territorio. En definitiva, la metodología empleada puede ser de gran utilidad para el apoyo a gobiernos nacionales o autonómicos en el establecimiento de estrategias para los territorios rurales, partiendo del conocimiento global de la dinámica interna de éstos. La comparación de los resultados obtenidos una vez las estrategias de desarrollo se lleven a cabo en las diversas comarcas de la comunidad andaluza serán objeto de nuevos análisis con los que validar las expectativas proporcionadas a los actores del territorio que elaboraron la estrategia de los territorios rurales, y en concreto la correspondiente a la comarca de Jerez.

\section{Agradecimientos}

Los autores desean agradecer a D. Francisco Alba, Director General de la Consejería de Agricultura y Pesca de la Junta de Andalucía, y a D. Juan Manuel Valderas y su equipo en la empresa Desarrollo Agrario y Pesquero (DAP) de la Consejería de Agricultura y Pesca de la Junta Andalucía por las facilidades dados. Igualmente desean agradecer a dos evaluadores externos por las indicaciones realizadas que sin duda han contribuido a la mejora de una versión previa del artículo.

\section{REFERENCIAS BIBLIOGRÁFICAS}

AMBROSIO-ALBALÁ, M. y DELGADO M.M. (2008): "Understanding rural areas dynamics from a complex perspective. An application of Prospective Structural Analysis". 12th Congress of the European Association of Agrarian Economists (EAAE), Gante, 26-29 agosto.

AMBROSIO-ALBALÁ, M. y BASTIAENSEN, J. (2010): "The new territorial paradigm of rural development: theoretical foundations from systems and institutional theories", IOB Discussion Paper Series, 2010/02. Amberes: Universidad de Amberes. 
ASTIGARRAGA, E. (2006): Consulta electrónica: duda para interpretar tasas de relleno. Blog en línea: http://www.eibar.org/blogak/prospektiba/196\#comments [Último acceso: 29 abril 2011]

ASTIGARRAGA, E. (blog): "El análisis estructural. Identificación y determinación de los factores de futuro para una empresa o un sector" Materiales de clase. http://www.prospectiva.eu/zaharra/02_Aestructural_Este.pdf [Último acceso: 29 abril 2011]

AXELROD, R. (1976): Structure of Decision: The Cognitive Maps of Political Elites. Princenton, New Jersey: Princeton University Press.

BOLANOS, R.; FONTELA, E., NENCLARES, A. and PASTOR, P. (2005): "Using interpretive structural modelling in strategic decision-making groups" en Management Decision, 43(6), pp. 877-895.

CARIOLA, M. y ROLFO, S.(2004): "Evolution in the rationales of foresight in Europe", en Futures, 36(10), pp.1063-1075.

COMISIÓN EUROPEA (1988): El futuro del mundo rural. COM (88) 501 end.

COMISIÓN EUROPEA (1991): LEADER I Comunicación a los Estados Miembros. OJEC C 73 de 19 de Marzo.

COATES, J.F. (2000): "Scenario planning" en Technological forecasting and social change, 65(1), pp. 115-123.

DE MIGUEL, F. y MANRESA, A. (2004): "Modelos SAM lineales y distribución de renta: una aplicación para la economía extremeña" en Estudios de Economía Aplicada, 22(3), Diciembre, pp. 577-603.

DELGADO, M., RAMOS, E. y PRETEL, A. (2007): "Nuevas tendencias en evaluación de programas de desarrollo rural territorial: un proceso de autoevaluación en el altiplano de Granada" en Revista Española de Estudios Agrosociales y Pesqueros, (213), pp. 11-40.

DIEZ, J.I. y GUTIERREZ, R.A. (2008): "La transformación de las políticas de desarrollo económico: de la planificación del Estado Nacional a la gestión de las economías locales" en Revista de Estudios Regionales, (83), pp. 111142.

FORRESTER, J.W. (1961): Industrial dynamics. Productivity Press.

GARCÍA NICOLAS, C. (2009): "El coste de la cohesión: la realidad de una Europa desigual" en Estudios de Economía Aplicada, 27(3), Diciembre, pp. 679-698.

GAVIGAN, J.P. y SCAPOLO F. (2001): "Prospectiva regional: comprobación futura y validación de las estrategias de desarrollo" en The IPTS Report, (59), noviembre. http://vlex.com/vid/prospectiva-comprobacion-validacion118774 [Último acceso: 29 abril 2011].

GERTLER, M.S. y WOLFE, D.A. (2004): "Local social knowledge management: Community actors, institutions and multilevel governance in regional foresight exercises" en Futures, 36(1), pp. 45-66. 
GODET, M. (1985): Prospectiva y Planificación Estratégica. París: CPE Economica.

GODET, M. (1993): De la anticipación a la acción. Manual de prospectiva y estrategia. Barcelona: Boixareus Editores.

GODET, M. (2001): Creating futures. Scenario planning as a strategic management tool, Londres: Economica-Brookings.

GODET, M. y BOURSE, F. (1989): MICMAC Ver.6.1.2. Paris: Laboratoire d'Investigation en Prospective, Stratégie et Organisation (LIPSOR). http://www.laprospective.fr/methodes-de-prospective/les-methodes/51micmac.html [Último acceso: 29 abril 2011]

GÓMEZ-LIMÓN, J.A. y GÓMEZ-RAMOS, A. (2008): "Análisis prospectivo de la agricultura en España" en Revista Económica de Castilla-La Mancha, (13), pp . 103-140

GÓMEZ-LIMÓN, J.A.; GÓMEZ-RAMOS, A. y SANCHEZ, G. (2009): "Foresight analysis of agricultura sector at regional level" en Futures, 41(5), pp. 313324.

GONZALEZ FERNANDEZ, M. (2006): "Idas y vueltas en el desarrollo rural. De la diversificación de las economías locales a los rural como categoría económica global", en Revista Española de Estudios Agrosociales y Pesqueros, (211), pp. 121-141.

GROSSMANN, I. (2006): "Three scenarios for the greater Hamburg region" en Futures, 38(1), pp. 31-49.

HABEGGER, B. (2010): "Strategic foresight in public policy: Reviewing the experiences of the UK, Singapore, and the Netherlands" en Futures, 42(1), pp. 49-58.

HAIR, J.F.; ANDERSON, R.E.; TATHAM, R.L. y BLACK, W.C. (1999): Análisis multivariante, $5^{\mathrm{a}}$ edición. Madrid: Prentice Hall.

JAIMES, D.; ALEXANDER, C. y NARVÁEZ, P. (2003): "Prospectiva de la producción de oleoquímicos derivados del aceite de palma y de palmiste en Colombia" en Revista Ingeniería e Investigación, (52) Julio, pp. 63-74.

JANES, F. (1988): "Interpretive structural modelling: a methodology for structuring complex issues" en Transactions of the Institute of Measurement \& Control, 10(3), pp. 145-154.

JUNTA DE ANDALUCÍA (2008): Nueva Estrategia Rural para Andalucía. El proyecto NERA, Consejería de Agricultura y Pesca. Sevilla: Ed. Junta de Andalucía.

KALLAS, Z. y GÓMEZ-LIMÓN, J.A., (2007): "Valoración de la multifuncionalidad agraria: una aplicación a través del método de los experimentos de elección". en Estudios de Economía Aplicada, 25(1), Abril, pp. 107-144.

KELLY, R. (2004): "Futures thinking to achieve sustainable development at local level in Ireland" en Foresight, 6(2), pp. 80-90. 
KINSELLA J.; COMMINS, P.; RIORDAN, B.; WALSH, J.A.; MEREDITH, D.; PITTS, E.; CARTON, O.T.; TUNNEY, H.; FINN, J.; DOWNEY, L.; FENNESSY, J.; LENNAN, L.M. y CREHAN, P. (2005): "Rural Ireland 2025" en The EFMN Foresight Brief, 60. Brussels: European Foresight Monitoring Network (EFMN).

KOSKO, B. (1986): "Fuzzy cognitive maps" en International Journal of ManMachine Studies, 24(1), pp. 65-75.

LENDARIS, G.G. (1980): "Structural Modeling: A tutorial guide" en IEEE transactions on systems, man and cybernetics, 10(12), pp. 807-840.

LLOP LLOP, M. (2007): "Descomposición de los cambios de multiplicadores en una matriz de contabilidad social: un ejemplo para la economía catalana" en Estudios de Economía Aplicada, 25(3), Abril, pp. 743-758.

MAHROUM, S. (2007): “England's Rural Futures Project: Scenario Creation \& Backcasting" en The EFMN Foresight Brief, 117. Brussels: European Foresight Monitoring Network (EFMN).

MINGERS, J. y ROSENHEAD, J. (2004): "Problem structuring methods in action" en European Journal of Operational Research, 152(3), pp. 530-554.

ROSENHEAD, J. (1996): "What's the problem? An introduction to problem structuring methods" en Interfaces, 26(6), pp. 117-131.

SARRIA, Y.; HERNÁNDEZ, P. y AGUILERA E. (2009): "Aplicación del análisis estructural para el estudio del ordenamiento territorial en el sistema de infraestructura técnica, caso de estudio" en Revista OIDLES, 3(7), diciembre.

SCHLANGE, L.E. y JÜTTNER, U. (1997): "Helping managers to identify the key strategic issues" en Long Range Planning, 30(5), pp. 777-786.

SUMPSI VIÑAS, J. M. (2006): "Experiencias de desarrollo rural con enfoque territorial en la Unión Europea y América Latina" en Revista española de Estudios Agrosociales y Pesqueros, (209), pp. 41-71.

VERGARA, P. y ARRAIS DE SOUZA, M. (2005): "Planificación turística en tiempos de incertidumbre", en Ciencias Sociales Online, II(2), Septiembre, pp. 65 - 83. http://www.uvm.cl/csonline/2005_2/pdf/planificacion_turistica.PDF [Último acceso: 29 abril 2011].

VON BERTALANFFY, L. (1968): General System theory: Foundations, Development, Applications. New York: George Braziller.

WARFIELD, J. (1974): "Toward interpretation of complex structural models" en IEEE Transactions on Systems, Man \& Cybernetics, 4(5), pp. 405-417.

WEIMER-JEHLE, W. (2006): "Cross-impact balances: A system-theoretical approach to cross-impact analysis" en Technological Forecasting and Social Change, 73(4), pp. 334-361. 
